\title{
Prolonged Mek1/2 suppression impairs the developmental potential of embryonic stem cells
}

Jiho Choi ${ }^{1,2,3,4 *}$, Aaron J. Huebner ${ }^{1,2,3,4 *}$, Kendell Clement ${ }^{3,4,5}$, Ryan M. Walsh ${ }^{1,2,3,4}$, Andrej Savol ${ }^{1}$, Kaixuan Lin ${ }^{6}$, Hongcang Gu ${ }^{5}$, Bruno Di Stefano ${ }^{1,2,3,4}$, Justin Brumbaugh ${ }^{1,2,3,4}$, Sang-Yong Kim ${ }^{7}$, Jafar Sharif ${ }^{8}$, Christopher M. Rose ${ }^{9}$, Arman Mohammad ${ }^{5}$, Junko Odajima ${ }^{2}$, Jean Charron ${ }^{10}$, Toshi Shioda ${ }^{2}$, Andreas Gnirke ${ }^{5}$, Steven Gygi ${ }^{9}$, Haruhiko Koseki ${ }^{8}$, Ruslan I. Sadreyev ${ }^{1}$, Andrew Xiao ${ }^{6}$, Alexander Meissner ${ }^{3,4,5}$ \& Konrad Hochedlinger ${ }^{1,2,3,4}$

Concomitant activation of the Wnt pathway and suppression of Mapk signalling by two small molecule inhibitors ( $2 \mathrm{i}$ ) in the presence of leukaemia inhibitory factor (LIF) (hereafter termed $2 \mathrm{i} / \mathrm{L}$ ) induces a naive state in mouse embryonic stem (ES) cells that resembles the inner cell mass (ICM) of the pre-implantation embryo ${ }^{1}$. Since the ICM exists only transiently in vivo, it remains unclear how sustained propagation of naive ES cells in vitro affects their stability and functionality. Here we show that prolonged culture of male mouse ES cells in $2 \mathrm{i} / \mathrm{L}$ results in irreversible epigenetic and genomic changes that impair their developmental potential. Furthermore, we find that female ES cells cultured in conventional serum plus LIF medium phenocopy male ES cells cultured in $2 \mathrm{i} / \mathrm{L}$. Mechanistically, we demonstrate that the inhibition of Mek1/2 is predominantly responsible for these effects, in part through the downregulation of DNA methyltransferases and their cofactors. Finally, we show that replacement of the Mek1/2 inhibitor with a Src inhibitor preserves the epigenetic and genomic integrity as well as the developmental potential of ES cells. Taken together, our data suggest that, although short-term suppression of Mek1/2 in ES cells helps to maintain an ICM-like epigenetic state, prolonged suppression results in irreversible changes that compromise their developmental potential.

Dysregulation of DNA methylation as well as of Wnt and Mapk signalling has been linked to cellular transformation and chromosomal instability in ES cells ${ }^{2-5}$. Therefore, we sought to determine whether sustained perturbation of the Wnt/Mapk pathway and associated DNA hypomethylation during $2 \mathrm{i} / \mathrm{L}$ culture affects the stability and functionality of ES cells. Specifically, we used three isogenic $129 \mathrm{~S} 6 \times \mathrm{C} 57 \mathrm{~B} 6 \mathrm{~F}_{1}$ male ES cell lines (J34, J35 and J37), which were derived in 2i/L medium and then cultured in serum plus LIF (S/L) medium for four additional passages (Fig. 1a). Each ES cell line was subsequently passaged onto a feeder layer of irradiated mouse embryonic fibroblasts (MEFs) in either $2 \mathrm{i} / \mathrm{L}$ or $\mathrm{S} / \mathrm{L}$ and propagated for an additional 6 or 16 passages (final passages p10 or p20, respectively). To assess the reversibility of any observed changes, we also switched the p20 2i/L-cultured ES cell lines back into S/L for an additional 3 or 10 passages.

We assessed global DNA methylation patterns in our cultured ES cells using reduced representation bisulfite sequencing (RRBS). Similar to previous reports ${ }^{6-8}$, global methylation levels of $2 \mathrm{i} / \mathrm{L}$-cultured ES cells were lower than those of S/L-cultured ES cells and became remethylated when switched back to S/L (Extended Data Fig. 1a-c). This demethylation and subsequent remethylation occurs across most methylated features including CpG islands, shores, short and long interspersed nuclear elements (SINEs and LINEs, respectively) and long terminal repeats (LTRs) (Extended Data Fig. 1d, e). By contrast, 2i/L culture caused a progressive and irreversible erosion of DNA methylation at most imprinting control regions (ICRs), resulting in the biallelic expression of the imprinted gene, Impact (Fig. 1b-d and Extended Data Fig. 1f, g). We confirmed biallelic expression of additional imprinted genes using a Mus musculus $\times$ Mus spretus $\mathrm{F}_{1}$ stem-cell line cultured in $2 \mathrm{i} / \mathrm{L}$ for six passages (Extended Data Fig. $1 \mathrm{~h}$ ).

Female ES cells cultured in S/L exhibit attenuated Mapk signalling, increased Wnt signalling, and upregulation of transcription factors associated with a naive-like state when compared to male ES cells cultured in $\mathrm{S} / \mathrm{L}^{9}$. Furthermore, female ES cells cultured in S/L were reported to be hypomethylated at imprinted and non-imprinted loci ${ }^{6,10,11}$, although the extent of hypomethylation seems to be variable $^{12}$. We therefore compared the methylation status of our male ES cells with three isogenic $\left(129 \mathrm{~S} 6 \times \mathrm{C} 57 \mathrm{BL} 6 \mathrm{~F}_{1}\right)$ female ES cell lines that were cultured in S/L for six passages. At the time of analysis, our female ES cell lines retained both X chromosomes, were hypomethylated globally and at ICRs, and biallelically expressed Impact (Fig. 1d-f and Extended Data Fig. 1i). Unsupervised clustering based on global methylation levels revealed that S/L-cultured female ES cells clustered with ICM cells and 2i/L-cultured male ES cells (Fig. 1e). However, when the same samples were clustered based on ICR methylation levels, female ES cells clustered with 2i/L-cultured male ES cells but apart from ICM cells and S/L-cultured male ES cells (Fig. 1f). Moreover, we noticed a substantial overlap between differentially methylated regions that distinguish male from female ES cells grown in S/L, and those that distinguish male ES cells grown in $\mathrm{S} / \mathrm{L}$ from those grown in $2 \mathrm{i} / \mathrm{L}$ (Fig. 1g). Thus, female ES cells cultured in $\mathrm{S} / \mathrm{L}$ resemble male ES cells cultured in $2 \mathrm{i} / \mathrm{L}$ with respect to imprinted and non-imprinted methylation patterns.

The genomic distribution pattern of the histone variant H2A.X in pluripotent stem cells has been shown to predict their developmental potential ${ }^{13}$. Because $2 \mathrm{i} / \mathrm{L}$ changes the chromatin landscape of ES cells relative to $\mathrm{S} / \mathrm{L}^{14}$, we performed chromatin-immunoprecipitation followed by sequencing (ChIP-seq) for H2A.X on 2i/L-cultured male ES cells and S/L-cultured female ES cells. We found that $2 \mathrm{i} / \mathrm{L}$-cultured male ES cells and S/L-cultured female ES cells lack H2A.X binding at 38,925 and 51,442 regions, respectively, relative to S/L-cultured male ES cells (Extended Data Fig. 2a-d). Moreover, genomic regions that lost H2A.X binding in both $2 \mathrm{i} / \mathrm{L}$-cultured male ES cells and S/Lcultured female ES cells (12,179 regions) were near genes associated with gastrulation, organ development and formation of the germ layer (Extended Data Fig. 2c-e). Of note, switching 2i/L-cultured male ES

\footnotetext{
${ }^{1}$ Massachusetts General Hospital Department of Molecular Biology, Boston, Massachusetts 02114, USA. ${ }^{2}$ Massachusetts General Hospital Cancer Center and Center for Regenerative Medicine, Boston, Massachusetts 02114, USA. ${ }^{3}$ Department of Stem Cell and Regenerative Biology, Harvard University, Cambridge, Massachusetts 02138, USA. ${ }^{4}$ Harvard Stem Cell Institute, 1350

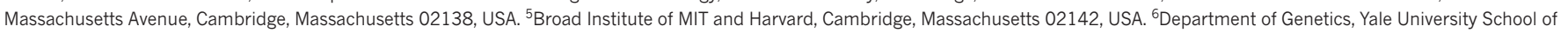

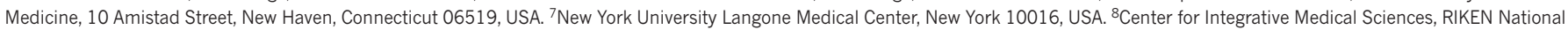
Research and Development Agency, 1-7-22 Suehiuro-cho, Tsurumi-ku, Yokohama-shi, Kanagawa-ken 230-0045, Japan. ${ }^{9}$ Department of Cell Biology, Harvard Medical School, 240 Longwood Avenue, Boston, Massachusetts 02115, USA. ${ }^{10}$ Centre de recherche sur le cancer de l'Université Laval, CRCHU de Québec, L'Hôtel-Dieu de Québec, 9 , rue McMahon, Quebec G1R 2J6, Canada. *These authors contributed equally to this work.
} 
a

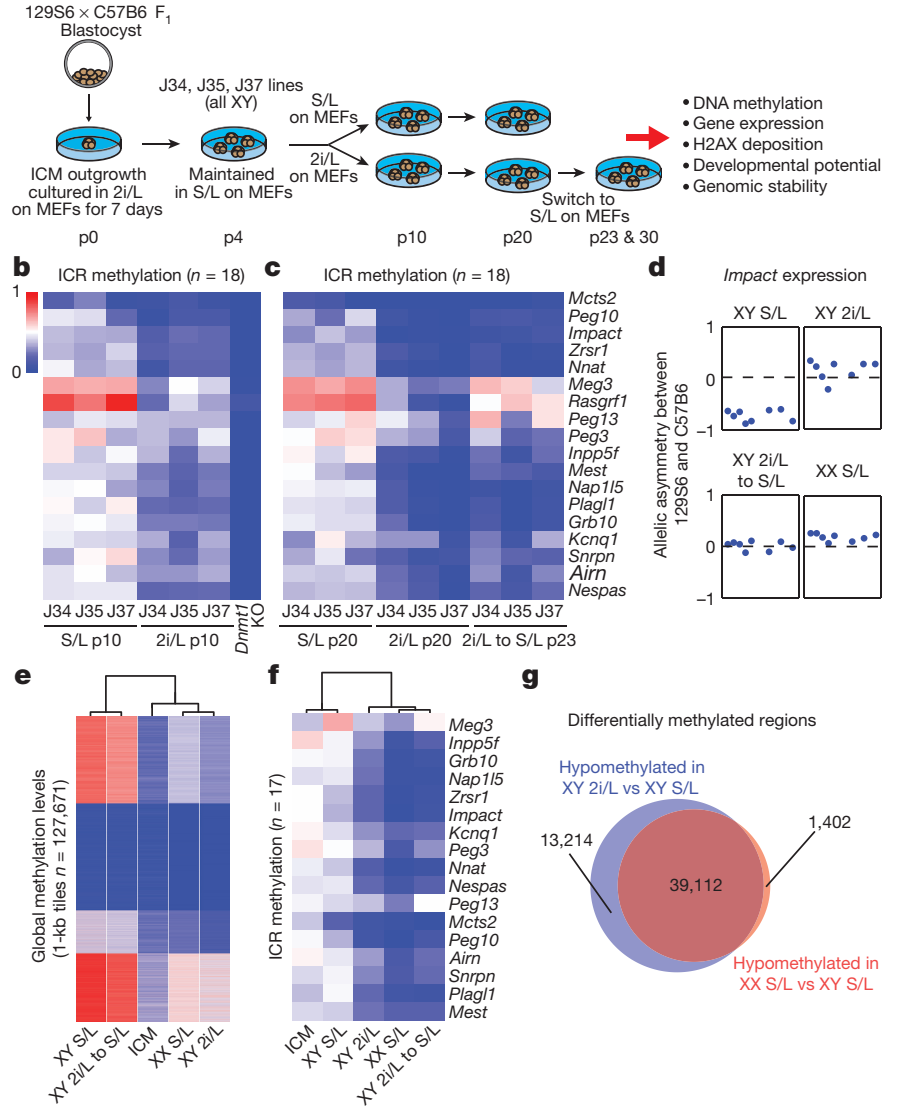

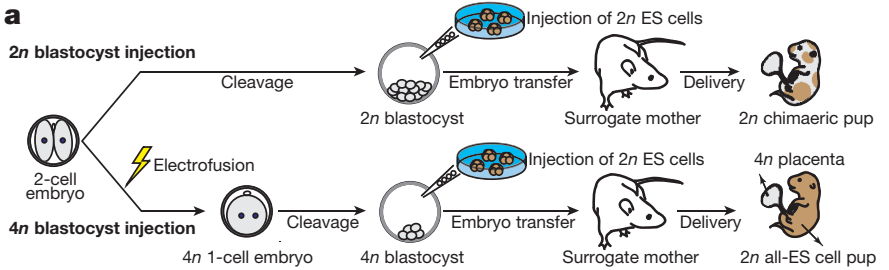

b

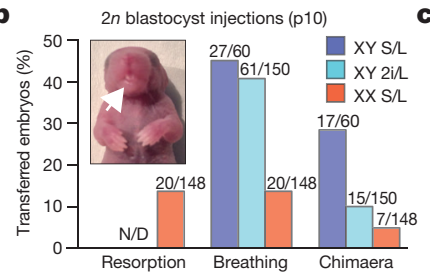

d
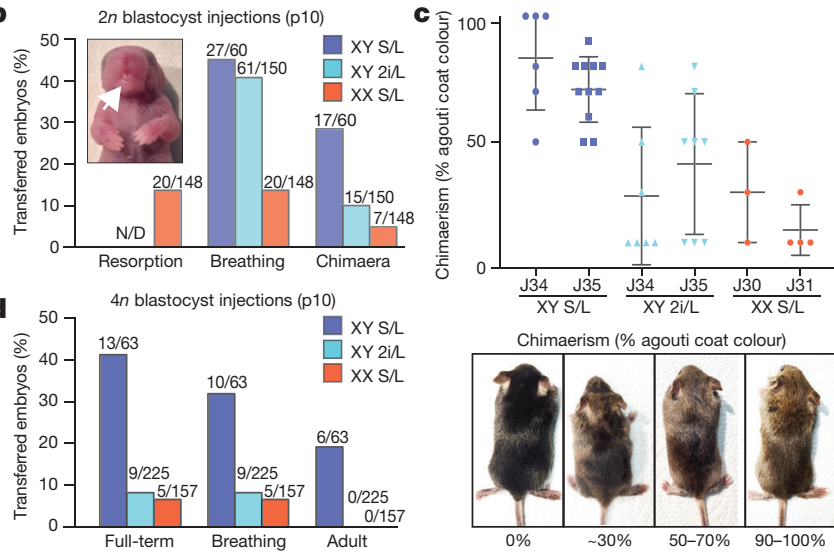

Chimaerism (\% agouti coat colour)

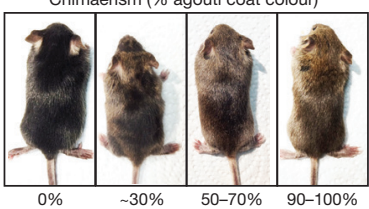

Figure 2 | Prolonged 2i/L culture impairs the developmental potential of ES cells. a, Schematic of blastocyst injection protocol. $\mathbf{b}, 2 n$ blastocyst injections. Numbers of animals obtained per total number of transferred embryos are shown. White arrow indicates the protruding tongue of a female pup. N/D, not determined. See Supplementary Table 1 for details. c, Top, quantification of coat colour chimaerism of the chimaeras from $\mathbf{b}$. Bottom, representative images of the varying degrees of chimaerism. d, $4 n$ blastocyst injections. Numbers of animals obtained per total number of transferred embryos are shown. See Supplementary Table 2 for details.

Figure 1 | Erosion of genomic imprints in $2 \mathrm{i} / \mathrm{L}$-cultured male ES cells and $\mathrm{S} / \mathrm{L}$-cultured female ES cells. a, Schematic of experimental design. p, passage. b, c, ICR methylation levels in ES cells at p10 (b) and p20 and p23 (c). KO, knockout. d, Allelic expression of the imprinted gene Impact. e, f, Global (e) and ICR (f) methylation levels in male ES cells at p20 and p23 ( $n=3$ biological replicates) and female ES cells at p6 $(n=3$ biological replicates). g, Overlap of differentially methylated regions.

cells to S/L did not restore H2A.X binding at most loci, suggesting that H2A.X depletion is irreversible at many sites, similar to ICR methylation (Extended Data Fig. 2a, b).

Considering that proper genomic imprinting and H2A.X deposition are essential for development ${ }^{13,15}$, we injected our different ES cell lines into $2 n$ or $4 n$ host blastocysts to determine their developmental potential (Fig. 2a and Supplementary Tables 1,2). We found that $2 \mathrm{i} / \mathrm{L}$ cultured male ES cells and S/L-cultured female ES cells gave rise to fewer pups than S/L-cultured male ES cells after $2 n$ blastocyst injections (Fig. 2b), and several of the pups we obtained displayed phenotypes associated with aberrant imprinting ${ }^{15}$ (Fig. 2b, inset, and Extended Data Fig. 3a). Moreover, adult chimaeras derived from $2 \mathrm{i} / \mathrm{L}$-cultured male ES cells or S/L-cultured female ES cells had far less agouti coat colour, indicating a poor contribution to adult tissues (Fig. 2c). Nevertheless, some of these low-grade chimaeras produced ES-cell-derived offspring, demonstrating their ability to produce functional germ cells (Extended Data Fig. 3b). When $4 n$ blastocyst injections were performed, $2 \mathrm{i} / \mathrm{L}$ cultured male ES cells and S/L-cultured female ES cells were 5-6 times less efficient than S/L-cultured male ES cells at producing entirely ES cell-derived (all-ES cell) pups (Fig. 2d and Extended Data Fig. 3c-e). These results are consistent with a previous report, which showed that early passage female ES cells are slightly less effective at producing all-ES cell pups compared to male ES cells ${ }^{16}$. Notably, none of the all-ES cell pups generated from $2 \mathrm{i} / \mathrm{L}$-cultured male or S/L-cultured female ES cells survived beyond birth (Fig. 2d and Extended Data Fig. $3 \mathrm{~d}$ ). Furthermore, we found that one of our male ES cell lines (J37) was unable to generate full-term all-ES cell pups when passaged in

2i/L for 16 passages (Supplementary Table 2). This ES cell line also failed to produce well-differentiated teratomas, suggesting that mutations acquired in this line compromised its differentiation potential (Extended Data Fig. 4a, b). On the basis of these data, we conclude that continual passaging of male ES cells in $2 \mathrm{i} / \mathrm{L}$ or female ES cells in S/L results in a progressive impairment of their developmental potential.

Perturbation of Wnt/Mapk signalling ${ }^{2,3}$, DNA hypomethylation ${ }^{4,5}$ and H2A.X loss $^{17}$ have been shown to affect genomic stability. We therefore performed karyotype analysis (Supplementary Table 3) and array comparative genomic hybridization (aCGH) on our ES cell lines cultured in $2 \mathrm{i} / \mathrm{L}$ or S/L. 2i/L-cultured ES cells at p10 and S/L-cultured ES cells at p20 had mostly diploid chromosome counts (40,XY) (Fig. 3a and Extended Data Fig. 5a). However, 2i/L-cultured ES cell lines at p20 exhibited recurrent chromosomal aberrations including trisomy 6, 8 and 19, as well as loss of the Y chromosome in many, if not all, cells analysed (Fig. 3a-c and Extended Data Fig. 5a). To exclude the possibility that these abnormalities are specific to our cell lines, we analysed a Rex1-GFP ES cell line ${ }^{18}$ that had been independently maintained in 2i/L conditions and found several chromosomal aberrations including trisomy 6 and 8 (Extended Data Fig. 5b). Although previous reports demonstrated that irradiated MEFs do not affect ES cells in a co-culture system $^{19}$, we also passaged one of our ES cell lines in $2 \mathrm{i} / \mathrm{L}$ without feeders for 16 passages. As expected, every cell karyotyped acquired trisomy 6 and 8 , confirming that the $2 \mathrm{i} / \mathrm{L}$-derived chromosomal aberrations in ES cells were not due to the presence of MEFs (Extended Data Fig. 5c). Considering these unexpected chromosomal abnormalities, we performed aCGH on DNA isolated from a rare full-term all-ES cell pup that was generated using p20 2i/L-cultured ES cells. We did not find evidence for gross copy number abnormalities in this pup (Extended Data Fig. 5d), even though the parental bulk cultures had chromosomal aberrations in every cell counted (Fig. 3a, middle, 3b). We surmise that rare euploid ES cells present in the culture experienced a strong positive selection during development, yet succumbed postnatally owing to the 


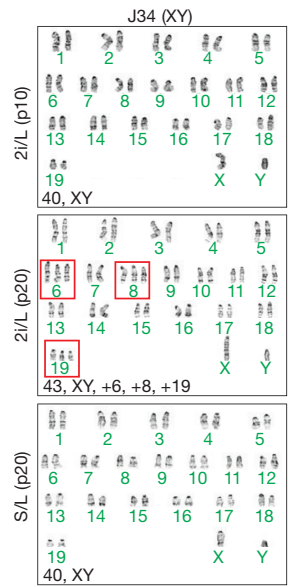

b

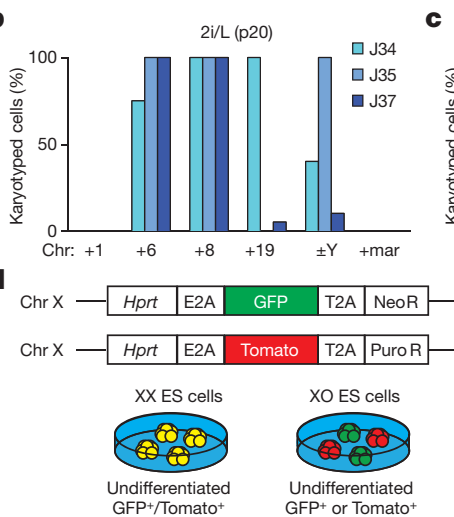

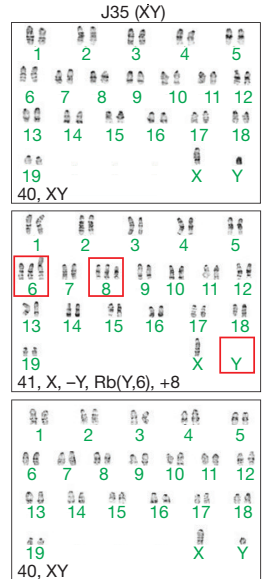

c

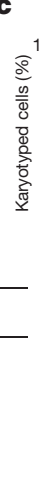

西

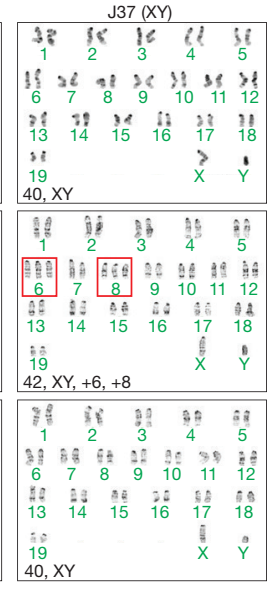

$\mathrm{S} / \mathrm{L}(\mathrm{p} 20)$

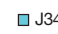

$\square \sqrt{35}$

$\square \mathrm{J} 37$
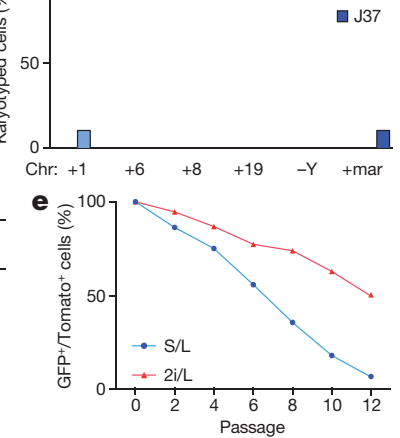

Figure 3 Impact of prolonged 2i/L culture on chromosomal stability. a, Karyotyping analysis of male ES cell lines. Red boxes indicate abnormal chromosomes detected. $\mathrm{Rb}(\mathrm{Y}, 6)$ denotes Robertsonian translocation between the $\mathrm{Y}$ chromosome and chromosome 6. b, c, Quantification of the chromosomal abnormalities found in $2 \mathrm{i} / \mathrm{L}$-cultured (b) and S/L-cultured (c) male ES cells at p20. + mar denotes chromosomal fragment of unknown origin. d, Schematic of the $\mathrm{X}^{\mathrm{G}} \mathrm{X}^{\mathrm{T}} \mathrm{ES}$ cell line and expected differences between XX and XO ES cells. e, Quantification of X chromosome loss in $\mathrm{X}^{\mathrm{G}} \mathrm{X}^{\mathrm{T}} \mathrm{ES}$ cells cultured in $\mathrm{S} / \mathrm{L}$ or $2 \mathrm{i} / \mathrm{L}$.

epigenetic aberrations present. To confirm this, we performed RRBS on explanted keratinocytes derived from chimaeric mice produced with 2i/L-cultured ES cells and found that they were indeed aberrantly hypomethylated at several imprinted loci (Extended Data Fig. 5e).

Female ES cells tend to lose one of their two X chromosomes with culture, thus becoming $\mathrm{XO}^{10}$. The loss of an X chromosome in XX ES cells has been associated with downregulation of naive-like genes and increased DNA methylation, similar to male ES cells cultured in $\mathrm{S} / \mathrm{L}^{9,11}$. We hypothesized that the naive-like state of XX female ES cellsparticularly as it relates to increased Wnt signalling and decreased Mapk signalling - is responsible for the selective pressure to lose an $\mathrm{X}$ chromosome. Thus, if the pharmacological inhibition of Gsk $3 \alpha / \beta$ and/ or Mek1/2 via 2i/L culture attenuates X chromosome loss in female ES cells, we would conclude that increased Wnt signalling and/or suppressed Mapk signalling in XX ES cells drives female ES cells to become $\mathrm{XO}$. To test this hypothesis, we developed a dual reporter female ES cell line $\left(\mathrm{X}^{\mathrm{G}} \mathrm{X}^{\mathrm{T}}\right)$ and measured $\mathrm{X}$ chromosome loss during $\mathrm{S} / \mathrm{L}$ and $2 \mathrm{i} / \mathrm{L}$ culture (Fig. $3 \mathrm{~d}$ and Extended Data Fig. 6a-c). Indeed, when we exposed $\mathrm{X}^{\mathrm{G}} \mathrm{X}^{\mathrm{T}} \mathrm{ES}$ cells to $2 \mathrm{i} / \mathrm{L}$, a substantial fraction of $\mathrm{X}^{\mathrm{G}} \mathrm{X}^{\mathrm{T}} \mathrm{ES}$ cells remained double-positive relative to $\mathrm{X}^{\mathrm{G}} \mathrm{X}^{\mathrm{T}} \mathrm{ES}$ cells cultured in S/L (Fig. 3e).

It remains unclear whether Wnt activation, Mapk suppression, or a combination of these activities is responsible for the epigenetic abnormalities we observed. We therefore performed RRBS on male ES cells cultured in S/L with one of the two inhibitors used in $2 \mathrm{i} / \mathrm{L}$ culture: the Mek1/2 inhibitor PD0325901 (S/L + PD) or the Gsk3 $\alpha / \beta$ inhibitor CHIR99021 (S/L + CHIR). We found that only ES cells exposed to S/L + PD acquired similar methylation levels to ES cells cultured in 2i/L (Fig. $4 \mathrm{a}$ and Extended Data Fig. 7a). To confirm these results, we analysed DNA methylation levels in male pluripotent stem cells deficient for both Mek1 and Mek2 (also known as Map2k1 and Map2k2, respectively) or for both $G s k 3 a$ and $G s k 3 b$. We found that the Mek double-knockout stem cells are hypomethylated globally and at ICRs whereas the Gsk3 double-knockout stem cells remain largely unchanged (Fig. 4b, c and Extended Data Fig. 7b). Notably, global methylation, including at ICRs, was further reduced in female ES cells cultured in S/L + PD when compared to female ES cells cultured in S/L (Extended Data Fig. 7c, d). Together, these data suggest that suppression of Mek $1 / 2$ is largely responsible for the observed epigenetic aberrations of $2 \mathrm{i} / \mathrm{L}$-cultured ES cells.

To understand how Mek1/2 inhibition induces global hypomethylation, we compared RNA sequencing (RNA-seq) and quantitative large-scale proteomics data generated from male ES cells cultured in either S/L or S/L + PD for three passages (Fig. 4d). Exposure of ES cells to $\mathrm{S} / \mathrm{L}+\mathrm{PD}$ resulted in an upregulation of transcription factors that have been associated with naive pluripotency (Extended Data Fig. 8a). Furthermore, all major DNA methyltransferases (Dnmt1, Dnmt $3 a$ and Dnmt3b) and associated cofactors (Dnmt3l and Uhrf1) were downregulated at the RNA and protein level in ES cells cultured in S/L + PD (Fig. 4e, Extended Data Fig. 8b and Supplementary Fig. 1). These observations are consistent with the previous notion that reduced levels of Uhrf1 and Dnmt3a/Dnmt3b underlie 2i/L-induced hypomethylation ${ }^{7,20}$.

We next tested whether suppression of Mek1/2 is sufficient to drive the chromosomal abnormalities we observed in male ES cells cultured in $2 \mathrm{i} / \mathrm{L}$. Karyotypes of our male ES cells cultured in S/L + PD for 16 passages revealed that every counted cell had some abnormality, contrary to ES cells exposed to S/L + CHIR (Extended Data Fig. 8c, d). To determine whether the genomic hypomethylation driven by Mek1/2 inhibition in ES cells is sufficient to select for the recurring karyotypic aberrations, we derived ES cells containing conditional triple-knockout alleles for Dnmt1, Dnmt3a and Dnmt3b. When we deleted the Dnmt genes and cultured the ES cells in S/L for 15 passages, we found that they remained euploid, suggesting that Mek1/2 suppression drives the selection for certain chromosomal abnormalities independently of DNA methylation (Extended Data Fig. 8e, f).

Considering the effect of continual Mek1/2 inhibition on ES cells, we determined whether previously reported alternative culture conditions $^{21,22}$ that do not inhibit Mek1/2 resemble naive ES cells while maintaining a normal developmental potential. We tested two defined media conditions supplemented with the PKC inhibitor Gö6983 and LIF (PKCi/L), or with the Src inhibitor CGP77675 in combination with CHIR and LIF (a2i/L) using our ES lines (Extended Data Fig. 9a). Although both $\mathrm{PKCi} / \mathrm{L}$ and $\mathrm{a} 2 \mathrm{i} / \mathrm{L}$ conditions were transcriptionally more similar to $\mathrm{S} / \mathrm{L}$ than $2 \mathrm{i} / \mathrm{L}$ conditions by RNA-seq analysis, a2i/L-cultured ES cells clustered with $2 \mathrm{i} / \mathrm{L}$-cultured ES cells when the ES cells were grouped using a previously published set of pluripotency and differentiation genes ${ }^{23}$ (Extended Data Fig. 9b-e). RRBS analysis revealed that ES cells cultured in $\mathrm{PKCi} / \mathrm{L}$ and a2i/L were globally hypermethylated and retained proper methylation of ICRs, thus preserving monoallelic expression of Impact (Fig. 4f-h and Extended Data Fig. 9f, g). The hypermethylation of a2i/L- and PKCi/L-cultured ES cells coincided with increased Dnmt3b and Uhrf1 protein levels relative to 2i/L-cultured ES cells (Fig. 4i and Supplementary Fig. 1). Furthermore, when we cultured our female $\mathrm{X}^{\mathrm{G}} \mathrm{X}^{\mathrm{T}} \mathrm{ES}$ cell line in $\mathrm{a} 2 \mathrm{i} / \mathrm{L}$, we observed an intermediate level of X chromosome loss when compared to culture in S/L + PD and S/L (Extended Data Fig. 9h). Collectively, these results suggest that replacement of the Mek1/2 inhibitor with a Src inhibitor in $2 \mathrm{i} / \mathrm{L}$ medium preserves a naive-like transcriptional state without affecting global and ICR methylation in ES cells.

We next assessed the developmental potential of PKCi/L- and a2i/Lcultured ES cells by $4 n$ blastocyst injections. Consistent with the preservation of normal ICR methylation patterns and a euploid karyotype 

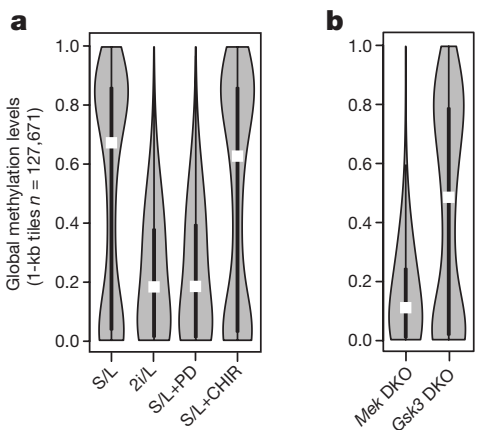

e

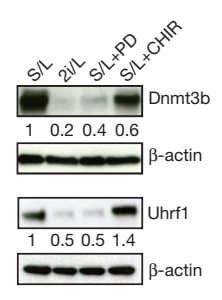

$\mathbf{f}$

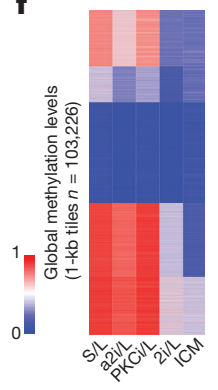

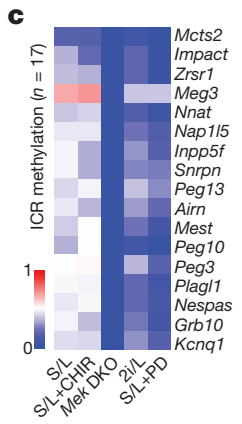

g

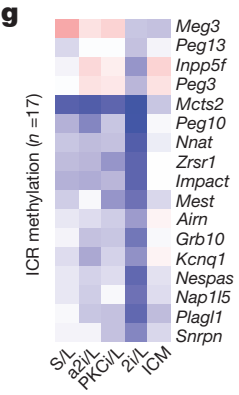

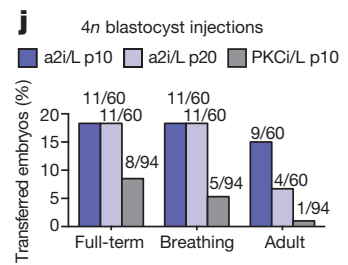

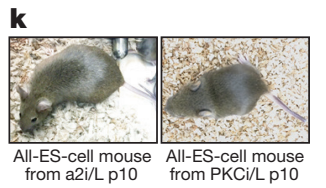

from a2i/L p10 $\quad$ from PKCi/L p10
Chromosome

I
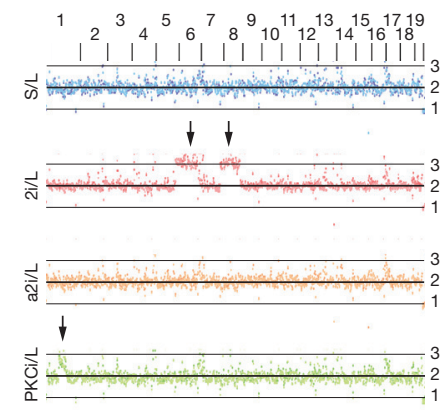

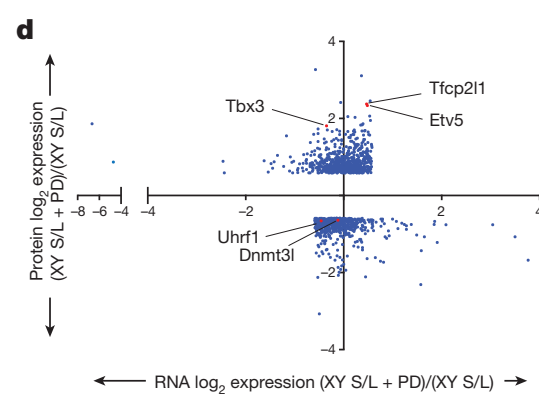

$\longleftarrow$ RNA $\log _{2}$ expression $(X Y S / L+P D) /(X Y S / L) \rightarrow$

h
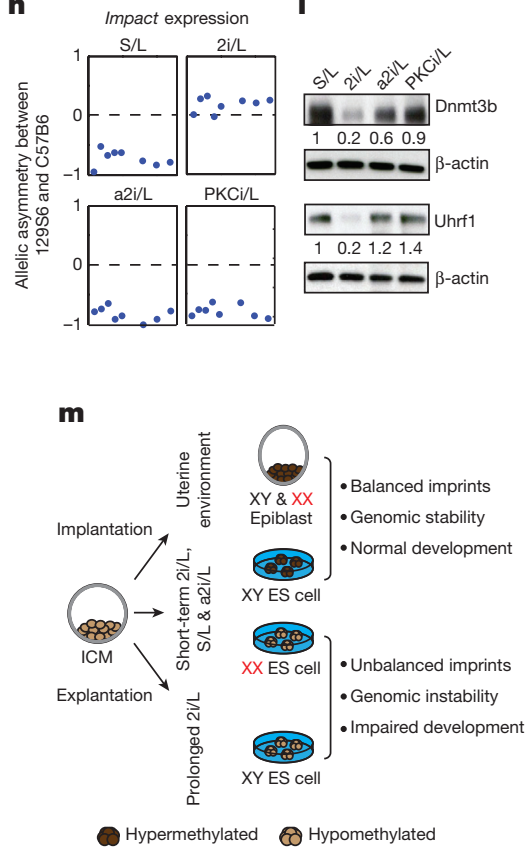

Figure $4 \mid$ Mek1/2 suppression underlies the epigenetic and chromosomal changes observed in ES cells. a, Global methylation levels of male ES cells cultured in indicated conditions $(n=3$ biological replicates). White dots, median value. $\mathbf{b}$, Global methylation levels of Mek1/2 double-knockout (DKO) iPS cells and Gsk3a/b double-knockout ES cells cultured in S/L. White dots, median value. c, ICR methylation levels in male pluripotent stem cells with the indicated pharmacological or genetic perturbations. d, Differential protein levels between ES cells cultured in $\mathrm{S} / \mathrm{L}(n=2$ technical replicates $)$ and $\mathrm{S} / \mathrm{L}+\mathrm{PD}(n=2$ technical replicates) $(>1.5$-fold) are plotted along the $y$ axis and the corresponding differentially expressed RNAs between ES cells cultured in S/L ( $n=3$ biological replicates) and S/L $+\mathrm{PD}(n=3$ biological replicates $)$ are plotted

under these conditions, we were able to generate several germlinecompetent, adult all-ES cell mice using ES cells cultured in a2i/L for 6 or 16 passages (Fig. 4j, k and Extended Data Fig. 9i-k). Unexpectedly, we only obtained one adult all-ES cell mouse using ES cells cultured in $\mathrm{PKCi} / \mathrm{L}$ for 6 passages (Fig. 4j, k), even though these ES cells were euploid and had normal ICR methylation levels (Extended Data Fig. 9k). While it remains unclear why PKCi/L-cultured ES cells generated so few all-ES cell pups and mice, it is possible that they acquired subtle genomic aberrations that compromised their developmental potential. In agreement with this notion, we detected a recurrent duplication on chromosome 1 in PKCi/L-cultured ES cells at p20 (Fig. 41 and Extended Data Fig. 9k).

Different conditions have recently been discovered that allow the propagation of naive human ES cells ${ }^{24,25}$. Similar to $2 \mathrm{i} / \mathrm{L}$-cultured mouse ES cells, naive human ES cells are globally hypomethylated, lose ICR methylation, and acquire karyotypic abnormalities ${ }^{26}$. Since inhibition of MEK1/2 via PD0325901 is common among current naive human culture conditions, we modified the naive medium ' 2 2iLGöY'25 along the $x$ axis. e, Western blot analysis of Dnmt3b and Uhrf1 in the labelled conditions. f, g, Global (f) and ICR (g) methylation levels in male ES cells cultured in indicated conditions. $\mathbf{h}$, Allelic expression of the imprinted gene Impact. i, Western blot analysis of Dnmt3b and Uhrf1 in the labelled conditions. $\mathbf{j}, 4 n$ blastocyst injections. Numbers of animals obtained per total number of transferred embryos are shown. See Supplementary Table 2 for details. k, Adult all-ES cell mice generated using a2i/L-cultured (left) or PKCi/L-cultured (right) male ES cells. 1, Chromosomal copy number analysis by whole-genome sequencing. Black arrows indicate trisomies 6 and 8 in 2i/L-cultured ES cell lines and the partial amplification of chromosome 1 in $\mathrm{PKCi} / \mathrm{L}$-cultured ES cell lines, respectively. $\mathbf{m}$, Graphical summary of results.

by replacing PD0325901 with the Src inhibitor used in a2i/L culture (termed a2iLGöY). We then monitored the maintenance of the naive state in human ES cells carrying a naive-specific $\triangle P E$-OCT4-eGFP (OCT4 is also known as POU5F1) reporter ${ }^{24}$ upon exposure to t2iLGöY or a2iLGöY. As expected, t2iLGöY maintained enhanced green fluorescent protein (eGFP) expression in most $\triangle$ PE-OCT4-eGFP ES cells. However, exposure of $\triangle$ PE-OCT4-eGFP ES cells to a2iLGöY led to a rapid loss of OCT4-eGFP expression, suggesting that the naive state cannot be maintained when SRC is inhibited instead of MEK1/2 (Extended Data Fig. 10a-c).

The $2 \mathrm{i} / \mathrm{L}$ culture system is undoubtedly a valuable approach to capture an ICM-like naive state ex vivo using pluripotent cells from different species and genetic backgrounds. However, our data demonstrate that extended culture of pluripotent cells in $2 \mathrm{i} / \mathrm{L}$ compromises their epigenetic and genomic stability as well as their developmental potential (Fig. $4 \mathrm{~m}$ ). Our results further demonstrate that attenuation of Mapk signalling via suppression of Mek1/2 is sufficient to induce genome-wide hypomethylation in ES cells, thus recapitulating the 
epigenetic state within the naive ICM. Inhibition of Src seems to maintain ES cells in an epigenetic state that lies in-between the naive ICM and the developmentally more advanced post-implantation epiblast. Considering that inhibition of Mek1/2 suppresses Mapk signalling to a greater extent than inhibition of $\mathrm{Src}^{22}$, our results indicate that the degree of Mapk signalling dictates the epigenetic state and chromosomal stability of ES cells. Comparing targets of Mek1/2 and Src will help us to understand how Mapk signalling facilitates the capture of different epigenetic states as well as drive the selection of particular chromosomal aberrations in pluripotent stem cells. These comparative studies should also provide valuable insights into the epigenetic regulation of early embryonic development and may lead to strategies to generate stable, human naive ES cells.

Online Content Methods, along with any additional Extended Data display items and Source Data, are available in the online version of the paper; references unique to these sections appear only in the online paper.

Received 13 June 2016; accepted 15 June 2017.

Published online 26 July; corrected online 9 August 2017 (see full-text HTML version for details).

1. Ying, Q.-L. et al. The ground state of embryonic stem cell self-renewal. Nature 453, 519-523 (2008)

2. Raggioli, A., Junghans, D., Rudloff, $S$ \& \& Kemler, R. $\beta$-catenin is vital for the integrity of mouse embryonic stem cells. PLoS One 9, e86691 (2014).

3. Chen, H. et al. Erk signaling is indispensable for genomic stability and self-renewal of mouse embryonic stem cells. Proc. Natl Acad. Sci. USA 112 E5936-E5943 (2015)

4. Chen, R. Z., Pettersson, U., Beard, C., Jackson-Grusby, L. \& Jaenisch, R. DNA hypomethylation leads to elevated mutation rates. Nature 395, 89-93 (1998).

5. Gaudet, F. et al. Induction of tumors in mice by genomic hypomethylation. Science 300, 489-492 (2003).

6. Habibi, E. et al. Whole-genome bisulfite sequencing of two distinct interconvertible DNA methylomes of mouse embryonic stem cells. Cell Stem Cell 13, 360-369 (2013).

7. Ficz, G. et al. FGF signaling inhibition in ES cells drives rapid genome-wide demethylation to the epigenetic ground state of pluripotency. Cell Stem Cell 13, 351-359 (2013).

8. Leitch, H. G. et al. Naive pluripotency is associated with global DNA hypomethylation. Nat. Struct. Mol. Biol. 20, 311-316 (2013).

9. Schulz, E. G. et al. The two active X chromosomes in female ES cells block exit from the pluripotent state by modulating the ES cell signaling network. Cell Stem Cell 14, 203-216 (2014).

10. Zvetkova, I. et al. Global hypomethylation of the genome in XX embryonic stem cells. Nat. Genet. 37, 1274-1279 (2005).

11. Choi, J. et al. DUSP9 modulates DNA hypomethylation in female mouse pluripotent stem cells. Cell Stem Cell 20, 706-719.e7 (2017).

12. Hackett, J. A. et al. Synergistic mechanisms of DNA demethylation during transition to ground-state pluripotency. Stem Cell Rep. 1, 518-531 (2013).

13. Wu, T. et al. Histone variant H2A.X deposition pattern serves as a functional epigenetic mark for distinguishing the developmental potentials of iPSCs. Cell Stem Cell 15, 281-294 (2014).

14. Marks, H. et al. The transcriptional and epigenomic foundations of ground state pluripotency. Cell 149, 590-604 (2012).

15. Plasschaert, R. N. \& Bartolomei, M. S. Genomic imprinting in development, growth, behavior and stem cells. Development 141, 1805-1813 (2014).

16. Nagy, A., Rossant, J., Nagy, R., Abramow-Newerly, W. \& Roder, J. C. Derivation of completely cell culture-derived mice from early-passage embryonic stem cells. Proc. Natl Acad. Sci. USA 90, 8424-8428 (1993).

17. Celeste, $A$. et al. H2AX haploinsufficiency modifies genomic stability and tumor susceptibility. Cell 114, 371-383 (2003).

18. Wray, J. et al. Inhibition of glycogen synthase kinase-3 alleviates Tcf3 repression of the pluripotency network and increases embryonic stem cell resistance to differentiation. Nat. Cell Biol. 13, 838-845 (2011).
19. Tamm, C., Pijuan Galitó, S. \& Annerén, C. A comparative study of protocols for mouse embryonic stem cell culturing. PLoS One 8, e81156 (2013)

20. Meyenn, von, F. et al. Impairment of DNA methylation maintenance is the main cause of global demethylation in naive embryonic stem cells. Mol. Cell 62, 848-861 (2016)

21. Dutta, D. et al. Self-renewal versus lineage commitment of embryonic stem cells: protein kinase C signaling shifts the balance. Stem Cells 29, 618-628 (2011).

22. Shimizu, T. et al. Dual inhibition of Src and GSK3 maintains mouse embryonic stem cells, whose differentiation is mechanically regulated by Src signaling. Stem Cells 30, 1394-1404 (2012).

23. Kolodziejczyk, A. A et al. Single cell RNA-sequencing of pluripotent states unlocks modular transcriptional variation. Cell Stem Cell 17, 471-485 (2015).

24. Theunissen, T. W. et al. Systematic identification of culture conditions for induction and maintenance of naive human pluripotency. Cell Stem Cell 15, 471-487 (2014)

25. Takashima, Y. et al. Resetting transcription factor control circuitry toward ground-state pluripotency in human. Cell 158, 1254-1269 (2014).

26. Pastor, W. A. et al. Naive human pluripotent cells feature a methylation landscape devoid of blastocyst or germline memory. Cell Stem Cell 18 323-329 (2016).

Supplementary Information is available in the online version of the paper.

Acknowledgements We thank members of the Hochedlinger laboratory for suggestions as well as L. Barrett for critical reading of the manuscript. Additionally, we thank A. Galvin, M. Gesner and M. Handley at the Massachusetts General Hospital flow cytometry core. We also thank A. L. Hawkins, S. Wang, A. Aggarwal and C. C. Morton at Brigham and Woman's Hospital CytoGenomics Core Laboratory for karyotyping analysis. We are grateful to K. Shioda from the Shioda laboratory for technical support for genome sequencing. We are also grateful to W. Yu at The Sidney Kimmel Cancer Center Microarray Core Facility at Johns Hopkins University for aCGH analysis, who was supported by $\mathrm{NIH}$ grant P30 CA006973 entitled Regional Oncology Research Center. We thank

T. Theunissen and R. Jaenisch for sharing human OCT4-eGFP ES cell lines and T. Graf for providing the Rex1-GFP ES cells. A.J.H. is supported by an American Cancer Society-New England Division-Ellison Foundation Postdoctoral Fellowship (PF-15-130-01-DDC). B.D.S. is supported by an EMBO long-term Fellowship (ALTF 1143-2015). T.S. is supported by NIH grant R21ES024861. A.Me. is a New York Stem Cell Foundation Robertson Investigator and supported by NIH grant 1P50HG006193 and the New York Stem Cell Foundation. K.H. was supported by funds from the MGH, HHMI, NIH (R01 HD058013-06) and the Gerald and Darlene Jordan Chair in Regenerative Medicine.

Author Contributions J. Choi and A.J.H. performed the bulk of the cell culture, teratoma assays, dot blot and flow cytometry analyses; J. Choi, A.J.H. and S.-Y.K. performed the blastocyst injections; J. Choi, A.J.H. and J. Charron generated the Mek1/2 double-knockout iPS cell lines; R.M.W. generated the $X^{G} X^{\top}$ ES cell line; J.S. and H.K. generated the Dnmt triple-knockout ES cell line; J. Choi, A.J.H. and J.B. conducted the western blot analysis; J.B., C.M.R. and S.G. performed the proteomics analysis; B.D.S. performed experiments related to human ES cell culture; J.O. and T.S. performed the genome sequencing analyses and analysed the RNA-seq data of interspecies iPS cell lines; K.C., H.G., A.Mo., A.G. and A.Me. performed the RRBS analyses; A.S. and R.I.S. performed the RNA-seq analyses; K.L. and A.X. conducted the H2A.X ChIP-seq analysis.; J. Choi, A.J.H. and K.H. designed the experiments, interpreted the results and wrote the manuscript.

Author Information Reprints and permissions information is available at www.nature.com/reprints. The authors declare no competing financial interests. Readers are welcome to comment on the online version of the paper. Publisher's note: Springer Nature remains neutral with regard to jurisdictional claims in published maps and institutional affiliations. Correspondence and requests for materials should be addressed to K.H. (khochedlinger@mgh.harvard.edu).

Reviewer Information Nature thanks T. Zwaka and the other anonymous reviewer(s) for their contribution to the peer review of this work. 


\section{METHODS}

Animal care and use. All mice used in the study were housed and bred in specific pathogen-free (SPF) rooms located in the AAALAC-accredited Center for Comparative Medicine vivarium at Massachusetts General Hospital. All mice were housed in ventilated cages on a standard $12 \mathrm{~h}$ light: $12 \mathrm{~h}$ dark cycle. All procedures involving mice adhered to the guidelines of the approved Massachusetts General Hospital Institutional Animal Care and Use Committee (IACUC) protocol number 2006 N000104.

ES cell derivation and cell culture. Blastocysts were isolated from the uterine horns of pregnant females at 3.5 days post coitum (d.p.c.) using a dissection microscope. Individual blastocysts, which for this study are considered as biological replicates, were plated onto feeder cells in 2i/L medium and cultured for 7 days. Emerging ICM outgrowths were directly picked into S/L medium on feeders to establish stable ES cell lines as previously reported ${ }^{11}$. Once lines were established, each line was genotyped to determine sex. ES cell lines were maintained by dissociating cells with $0.25 \%$ trypsin every 4 days and re-plating them onto irradiated MEFs. S/L medium contains knockout DMEM (Invitrogen) supplemented with $15 \%$ FBS (Hyclone), $1 \mathrm{mM} \mathrm{L}$-glutamine, $100 \mu \mathrm{M}$ non-essential amino acids (Invitrogen), $1 \times$ penicillin-streptomycin (Invitrogen), $0.1 \mathrm{mM} \beta$-mercaptoethanol (Invitrogen), and $10^{3} \mathrm{IU}$ LIF (in house). 2i/L medium contains a 1:1 mixture of DMEM/F12 supplemented with N2 (Invitrogen) and Neurobasal media with glutamine (Invitrogen) supplemented with B27 (Invitrogen), $1 \times$ penicillinstreptomycin (Invitrogen), $10^{3}$ IU LIF (in house), $1 \mu \mathrm{M}$ PD0325901 (Sigma) and $3 \mu \mathrm{M}$ CHIR99021 (Tocris). a2i/L medium contains a 1:1 mixture of DMEM/F12 supplemented with N2 (Invitrogen) and Neurobasal media with glutamine (Invitrogen) supplemented with B27 (Invitrogen), $1 \times$ penicillin-streptomycin (Invitrogen), $10^{3} \mathrm{IU}$ LIF (in house), $1.5 \mu \mathrm{M}$ CGP77675 (Tocris) and $3 \mu \mathrm{M}$ CHIR99021 (Tocris). $\mathrm{PKCi} / \mathrm{L}$ medium contains a 1:1 mixture of DMEM/F12 supplemented with N2 (Invitrogen) and Neurobasal media with glutamine (Invitrogen) supplemented with B27 (Invitrogen), $1 \times$ penicillin-streptomycin (Invitrogen), $10^{3}$ IU LIF (in house) and $5 \mu \mathrm{M}$ Gö6983 (Tocris). Irradiated MEFs (MTI-GlobalStem) were used throughout the study to ensure consistent conditions, unless noted. All lines were periodically tested for mycoplasma using the Lonza mycoalert kit and found to be negative. A detailed protocol describing the derivation and culture of mouse embryonic stem cells in the various conditions has been deposited at Protocol Exchange ${ }^{27}$.

iPS cell generation. Mek1 $1^{f l} ; M_{e k} 2^{-/-}$or $\mathrm{F}_{1}$ Mus musculus $\times$ Mus spretus MEFs were transduced with lentiviruses that facilitate the constitutive expression of M2rtTA and inducible expression of Oct4, Klf4, Sox 2 and c-Myc ${ }^{28}$. Infected MEFs were plated onto irradiated MEF feeders in S/L media supplemented with $2 \mu \mathrm{g} \mathrm{ml}^{-1}$ doxycycline until iPS cell colonies emerged. Doxycycline-independent, clonal iPS cells were picked and expanded. To remove the floxed Mek1 allele to generate Mek1/2 double-knockout iPS cells, we transiently transfected via electroporation a plasmid driving Cre recombinase and subcloned. Mek1/2 double-knockout iPS cells were verified by western blot and maintained on irradiated MEF feeders in S/L.

Generation of $D n m t 1 / 3 a / 3 b$ triple-knockout ES cells. Dnmt conditional triple-knockout ES cells were generated as described using blastocysts obtained from a Dnmt $1^{f l f l}, D n m t 3 a^{f l f l}$ and $D n m t 3 f^{f l f l}$ intercross $s^{4,29,30}$. To remove the floxed alleles, we transiently transfected via electroporation a plasmid driving Cre recombinase and subcloned. Dnmt triple-knockout ES cells were verified by western blot and maintained on irradiated MEF feeders in S/L.

RRBS. Genome-wide base-pair-level methylation was assayed using RRBS ${ }^{31}$ In brief, genomic DNA was digested using the MspI restriction enzyme and subjected to bisulfite treatment, which converts unmethylated cytosine residues to uracil. Genomic fragments were sequenced using next-generation sequencing, and aligned to the $\mathrm{mm} 9$ reference genome. Methylation levels of individual CpGs were measured by observing bisulfite conversion in the aligned read as compared to the reference genome. On average, 1.596 million $\mathrm{CpGs}$ were covered per sample, at a mean depth of 14.31 reads per CpG.

Region-level methylation values were computed by pooling coverage-weighted methylation levels in CpGs that were measured by at least 3 reads in $80 \%$ of samples. A consensus set of regions was defined using regions that had at least 5 reads in every sample. Genomic methylation plots used methylation levels of 127,671 non-overlapping $1-\mathrm{kb}$ tiles. Heat maps show methylation levels of 1-kb tiles clustered using $k$-means clustering $(\mathrm{k}=5)$, and samples clustered by hierarchical clustering. Promoters were defined as 1-kb up- and downstream from RefSeq transcription start sites. LINEs, SINEs, LTRs and IAPs were defined using RepBase. ICRs were defined as a set of 17 canonical ICRs covered by RRBS described previously ${ }^{32}$.

Violin plots were created using the 'vioplot' R package. The white dot represents the median. The box extends from the twenty-fifth to the seventy-fifth percentile of the data (the interquartile range). Whiskers extend from the box to the most extreme data point, which is no more than 1.5 times the interquartile range. Heat maps were created using the 'pheatmap' $\mathrm{R}$ package and clustering was performed using euclidean distance and complete linkage.

RNA-seq and analysis. RNA-seq libraries were constructed from RiboZeroselected RNA using NEBNext Ultra Directional RNA Library Prep Kit for Illumina (New England Biolabs) and sequenced on an Illumina HiSeq2500 instrument. Transcriptome mapping was performed with STAR ${ }^{33}$ version 2.3.0 using the Ensembl 67 release exon/splice-junction annotations. Approximately $55-90 \%$ of reads mapped uniquely. Read counts for individual genes were produced using the unstranded count feature in $\mathrm{HTSeq}^{34}$ version 0.6.0. Differential expression analysis was performed using the exactTest routine of the edgeR package ${ }^{35}$ after normalizing read counts and including only those genes with counts per million (c.p.m.) $>1$ for one or more samples ${ }^{36}$. Differentially expressed genes were defined based on the criteria of more than twofold change in expression value and false discovery rate $(\mathrm{FDR})<0.05$. Allelic expression asymmetry was quantified via samtools mpileup at exonic SNP sites that differentiated C57B6 from 129S6 reference genomes (indels excluded) ${ }^{37}$. To measure allelic asymmetry in expression, we used the asymmetry metric calculated as the ratio (129S6 - C57B6)/ (129S6 + C57B6), in which 129S6 and C57B6 are the numbers of reads assigned at a given SNP to the 129S6 and C57B6 alleles, respectively. This measure of allelic asymmetry ranges from -1 (C57B6 only) to 1 (129S6 only), with 0 corresponding to fully balanced biallelic expression.

RNA-seq and analysis of interspecific iPS cells. Total RNA was isolated from interspecific iPS cells using the RNeasy RNA-isolation kit (Qiagen), and their integrity (RNA integrity numbers (RIN) $\geq 9.4$ ) was confirmed using Agilent Tapestation with RNA High Sensitivity tapes. Illumina sequencing libraries with 6-bp single index were constructed from $1 \mu \mathrm{g}$ total RNA using the TruSeq Standard Total RNA LT kit with the Ribo-Zero Gold ribosomal RNA depletion module (Illumina). Libraries were subjected to size distribution analysis (200-500 bp, peaking at 280-290 bp) using Tapestation with the High Sensitivity D1000 tapes and quantified using the Illumina library qPCR quantification kit (KAPA Biosystems). Equal concentrations ( $4 \mathrm{nM}$ ) of libraries were pooled and subjected to Illumina NextSec deep sequencing using High Output Sequencing Kit version 2 (paired-end, 75 nucleotides +75 nucleotides). The fastq format raw reads data files were generated by the Illumina BaseSpace Sequencing Hub and subjected to pre-alignment quality control using the fastQC software (Babraham Institute). The fastq reads were aligned to the GRCm38/mm10 Mus musculus musculus (C57BL/6) reference genome sequence using the STAR aligner (version 2.4.2.a) with the UCSC annotation GTF file and the sjdbOverhang parameter 74 for genome indexing and alignment. The aligned reads were sorted and subjected to duplicates removal using the sambamba ${ }^{38}$ implementation of SAMtools ${ }^{39}$, resulting in $82-95$ uniquely mapped reads per RNA specimen. These reads were assigned to $\mathrm{mm} 10$-annotated genes and counted using the Bioconductor package Rsubread ${ }^{34}$ (69.1-71.2\% successfully assigned reads), and the gene counts were normalized to generate the c.p.m. read values using the negative binominal distribution model implemented by edge $\mathrm{R}^{36}$ Tracks of the uniquely mapped reads were directly inspected using the Integrative Genomics Viewer ${ }^{40}$, which reports paternal (M. spretus) and maternal (mm10 Mus musculus musculus) allelic counts for each SNP. Statistical significance of allelic counts at multiple SNPs in each gene were examined by $t$-test (unpaired, two-tailed, homoscedastic assumption).

Whole-genome sequencing. Genomic DNA $(1 \mu \mathrm{g})$ was sonicated using a Covaris S2 sonicator (Covaris) to approximately 350-bp peak fragments. To avoid PCR-derived bias of read counts, gDNA fragments were subjected to PCR-free synthesis of Illumina NextSeq deep sequencing libraries with the i7 index using the TruSeq DNA PCR-free LT Sample Preparation Kit - Set A (Illumina). Libraries were quantified using KAPA Illumina library quantification qPCR kit (KAPA Biosystems), and equal concentrations ( $4 \mathrm{nM}$ ) of libraries were pooled. Size distributions of gDNA fragments and deep sequencing libraries were examined using Agilent Tapestation with Genomic DNA screening tapes (Agilent Technologies) Single-read deep sequencing (76 nucleotides) was performed using Illumina NextSeq 500 sequencer with the high output v2 kit. Fastq files were subjected to a quality control process using Trim Galore! and fastQC (Babraham Institute), which excluded raw quality reads (Phred score $<20$ ) and deleted the Illumina adaptor sequences and the last one nucleotide at the $3^{\prime}$ end to avoid nucleotide base biases. The trimmed/QCed fastq reads were aligned to the mouse GRCm38/ mm10 reference genome sequence using the STAR aligner (version 2.4.2.a) with the genomic DNA alignment configuration, generating the bam format aligned reads files. The reads were extracted using the sambamba toolkit ${ }^{38}$. Numbers of the uniquely mapped reads were 46.2-59.6 million per sample, which corresponded to about 1.2-1.6 $\times$ average depth of genome coverage. Reads assigned to each chromosome are counted to generate $\mathrm{cnt}$ files. The chromosome count data were subjected to ploidy analysis, as previously described ${ }^{41}$. 
Native ChIP. Native ChIP assays were performed as previously described ${ }^{42}$. In brief, approximately 10 million ES cells were used for each ChIP and massive parallel sequencing (ChIP-seq) experiment. Chromatin pellets were briefly digested with MNase (New England BioLabs) and the mononucleosomes were monitored by electrophoresis $0.5 \mu \mathrm{g}$ anti-H2A.X antibody (active motif 39689) was used per ChIP experiment. Co-purified DNA and whole-cell extraction input genomic DNA were subject to library construction, cluster generation and next-generation sequencing (Illumina Genome Analyzer II and HiSeq 2000). The output sequencing reads were filtered and pre-analysed with Illumina standard workflow. After filtration, the qualified tags (in fastq format) were aligned to the mouse genome (mm9) with bowtie2 $(2.0 .6)^{43}$. Reads mapped to sex chromosomes were discarded. Aligned reads were used for peak calling using the RSEG algorithm (0.4.8) in mode 3 to identify differential binding regions relative to J34 S/L samples ${ }^{44}$. For cells cultured in $2 \mathrm{i} / \mathrm{L}$ and then switched back to serum, the combination of all male $\mathrm{S} / \mathrm{L}$ samples were used as control to reach a comparable throughput. The relative decreased regions were assigned to whole genome-wide 20 -kb windows to calculate a weighted average score and used for enumerating overlapping regions. Heat map and unsupervised hierarchical clustering analyses were drawn in 500-kb domains. GREAT was used to predict the putative target genes statistical relevance of associations between genomic regions and annotations ${ }^{45}$.

Blastocyst injections. Blastocyst ( $2 n$ and $4 n$ ) injections were performed as previously described ${ }^{46}$. In brief, female BDF1 mice were superovulated by intraperitoneal injection with pregnant mare serum (PMS) and human chorionic gonadotropin (hCG) and mated to BDF1 stud males. Zygotes were isolated from females with a vaginal plug $24 \mathrm{~h}$ after hCG injection. For $2 n$ injections, zygotes were cultured in vitro for 3 days in KSOM medium, and resultant blastocysts were injected with 10-15 ES cells before transfer into pseudopregnant recipient females. When newborn pups were not delivered until day 18 after the transfer, resorbed embryos were counted from the uteri of the recipient females and counted as 'resorption'. Surviving neonates with regular breathing were termed 'breathing', and two weeks later their chimaerism was assessed based on their agouti coat colour contributions. A sample size calculator, along with our own experience and previously published rates of chimaerism were used to determine the number of embryos injected and transferred.

For $4 n$ injections, zygotes were cultured overnight until they reached the 2-cell stage, at which point they were electrofused. After $1 \mathrm{~h}$, fused 1-cell embryos were carefully identified and separated from embryos that had failed to fuse, cultured in KSOM for another 2 days, and then injected with 15-20 ES cells before embryo transfer. C-section was performed at day 17 after the embryo transfer and embryos were termed 'full-term', 'breathing' and 'adult' when they reached the full-term, established regular breathing after birth and survived past 5 weeks, respectively. A sample size calculator, along with our own experience and previously published percentages obtaining all-ES cell mice were used to determine the number of embryos injected and transferred.

Teratoma assay. ES cells were grown to confluency in a single well of a confluent six-well plate. Cells were then trypsinized and counted. $2 \times 10^{6} \mathrm{ES}$ cells were resuspended in $300 \mu \mathrm{l}$ of ES cell media and injected subcutaneously into the flanks of a $n u / n u$ immunocompromised mouse. Teratomas were monitored and removed once tumour size reached $1 \mathrm{~cm}$ or tumours became ulcerated. Tumours were then weighed and processed for haemaytoxylin and eosin staining analysis. The tumour sections were blindly scored for the presence of each germ layer.

aCGH. Genomic DNA was isolated from frozen cell pellets using DNeasy tissue kit (Qiagen) following the manufacturer's protocol. Concentration and quality of isolated genomic DNA was assessed using a spectrophotometer (NanoDrop Technologies) to assure absence of contaminating protein $\left(A_{260 \mathrm{~nm}} / A_{280 \mathrm{~nm}}\right.$ ratio is greater than or equal to 1.8) and absence of carbohydrate, lipid and residual phenol $\left(A_{260 \mathrm{~nm}} / A_{230 \mathrm{~nm}}\right.$ ratio greater than or equal to 2.0$)$. Integrity of genomic DNA was confirmed by low voltage $0.6 \%$ agarose gel electrophoresis with mean band size of about $50 \mathrm{~kb}$. Samples were blinded and labelled according to Agilent's recommendation for aCGH. In brief, $1.5 \mu \mathrm{g}$ of genomic DNA was digested with $5 \mathrm{U}$ Alu $\mathrm{I}$ and Rsa I (Promega) for $2 \mathrm{~h}$ at $37^{\circ} \mathrm{C}$. Labelling reactions were carried out with total amount of fragmented DNA for $3 \mathrm{~h}$ at $37^{\circ} \mathrm{C}$ using a BioPrime Array CGH Genomic Labelling Module (Invitrogen) with $3 \mu \mathrm{mol}$ Cy5-dUTP or Cy3-dUTP (Perkin Elmer). Labelled samples were purified, concentrated on a Centricon YM-30 column (Millipore), and then mixed with $10 \times$ blocking agent and $2 \times$ hybridization buffer (Agilent Technologies). Samples were then hybridized on a SurePrint G3 Mouse $4 \times 180$ K CGH Microarray chip that contains 170,305 distinct biological probes taken from the mouse genome. Hybridized arrays were imaged with Agilent G2565BA microarray scanner using default settings. Data were extracted and analysed using Agilent's Genome Workbench software. Aberrant regions (gains or losses) were identified using a build-in ADM-2 algorithm with aberration filter setting as minimum number of probes for gains or losses $\geq 20$ and absolute $\log _{2}$ ratio for gains or losses $\geq 0.35$.
Karyotyping. Mouse ES cell lines were prepared for cytogenetic analysis by treatment with colcemid (Irvine Scientific) at a final concentration of $0.1 \mu \mathrm{g} \mathrm{ml}^{-1}$ for $3 \mathrm{~h}$ to accumulate cells in metaphase. Cells were then exposed to $0.075 \mathrm{M} \mathrm{KCl}$ for $25 \mathrm{~min}$ at $37^{\circ} \mathrm{C}$ and fixed with 3:1 methanol:acetic acid. Air-dried slides were generated and G-banded following standard GTG banding protocols. Two levels of blind analysis were pursued: for all lines, a minimum of 10 metaphase cells were counted, and 2-4 karyotypes prepared. For lines with a normal or consistent straightforward result, no further analysis was done. For lines with a complex karyotype, additional metaphases were counted (total 20), and 10 karyotypes prepared to better characterize the variability.

Generation of $\mathbf{X}^{\mathrm{G}} \mathbf{X}^{\mathrm{T}} \mathrm{ES}$ cell line. $\mathrm{X}^{\mathrm{G}} \mathrm{X}^{\mathrm{T}}$ lines were generated by CRISPR-Casmediated gene targeting in a newly derived $129 \mathrm{SvJ} \times \mathrm{C} 57 \mathrm{~B} 6 \mathrm{~F}_{1} \mathrm{ES}$ cell line. Two homology constructs were designed to integrate either E2A-GFP-T2A-Neo ${ }^{\mathrm{R}}$ STOP or E2A-Tomato-T2A-Puro ${ }^{\mathrm{R}}$-STOP in frame with the last exon of Hprt. The constructs were generated via PCR amplification and Giboson assembly (New England Biolabs) of the respective fluorescent protein and resistance marker flanked on either end by $500 \mathrm{bp}$ of homology to the Hprt locus. A single-guide RNA (sgRNA) was designed targeting the last exon of Hprt. Both homology constructs, the sgRNA and a construct containing Cas 9 were cotransfected into early passage female ES cells with Lipofectamine 2000 (Thermo Fisher). Following transfection, dual selection with G418 and puromycin was applied for 10-14 days. Resistant clones were picked and targeting was confirmed by Southern blot.

Dot blot analysis for $5 \mathrm{mC}$. Genomic DNA samples were prepared with twofold serial dilutions in TE buffer and then denatured in $0.4 \mathrm{M} \mathrm{NaOH} / 10 \mathrm{mM}$ EDTA at $95^{\circ} \mathrm{C}$ for $10 \mathrm{~min}$ and followed by adding an equal volume of cold $2 \mathrm{M}$ ammonium acetate ( $\mathrm{pH}$ 7.0). Denatured DNA samples were spotted on a nitrocellulose membrane in an assembled Bio-Dot apparatus (Bio-Rad) according to the manufacturer's protocol. The membrane was washed with $2 \times$ SSC buffer and UV-crosslinked for $10 \mathrm{~min}$. The membrane was then blocked with $5 \%$ non-fat milk for $1 \mathrm{~h}$ and incubated with an anti-5mC antibody (Active Motif; 39649), washed, and then incubated with a horseradish peroxidase (HRP)-conjugated secondary antibody. Chemiluminescence was measured, and subsequently stained with methlyene blue to detect the quantity of DNA loaded. Quantification was performed by Image-J software analysis.

Mass spectrometry analysis. Mass spectrometry analyses were performed on an Orbitrap Fusion Lumos mass spectrometer (ThermoFisher Scientific) coupled to an Easy-nLC 1200 ultra-high pressure liquid chromatography pump (ThermoFisher Scientific). Peptides were separated at $300 \mathrm{nl} \mathrm{min}{ }^{-1}$ using a selfpacked analytical column ( $75 \mu \mathrm{m}$ inner diameter) that was packed with $0.5 \mathrm{~cm}$ of Magic $\mathrm{C} 18$ resin $(5 \mu \mathrm{m}, 100 \AA$, Michrom Bioresources) followed by $35 \mathrm{~cm}$ of Sepax Technologies GP-C18 resin $(1.8 \mu \mathrm{m}, 120 \AA)$. Liquid chromatography buffers consisted of $0.1 \%$ formic acid (buffer $\mathrm{A}$ ) and $80 \% \mathrm{ACN}$ with $0.1 \%$ formic acid and liquid chromatography gradients were optimized to ensure equal elution of peptides throughout the analysis. Intact mass analysis of peptides (MS1) was performed in the Orbitrap (AGC target $1 \times 10^{6}, 120,000$ resolution, 100 ms maximum injection time) and used to select the 10 most abundant features for MS/MS (MS2) analysis. Candidate peaks were filtered based on charge sate $>2$ and monoisotopic peak assignment, and dynamic exclusion ( $60 \mathrm{~s} \pm 7.5$ p.p.m.) was enabled. Additionally, only one charge state was selected for each precursor for analysis of non-phosphorylated peptides. Each precursor was isolated with using a quadrupole mass filter $(0.5 \mathrm{Th}$ width) and then fragmented with collision-induced dissociation (CID, $35 \mathrm{NCE}$ ) ion the ion trap with distinct settings for non-phosphorylated (AGC target $=2.5 \times 10^{4}$, maximum injection time $=200 \mathrm{~ms}$ ) peptides. To alleviate the effects of precursor ion interference multiple fragment ions were isolated using synchronous precursor selection (SPS) before HCD (55 NCE, SPS notches $=8$, AGC target $=2.2 \times 10^{5}$, maximum injection time of $150 \mathrm{~ms}$ for non-phosphorylated peptides) MS3 fragmentation and Orbitrap analysis (50,000 resolution)

Mass spectrometry data analysis. A compilation of in-house software was used to convert mass spectrometric data (Thermo 'raw' files) to mzXML format, as well as to correct monoisotopic $m / z$ measurements and erroneous peptide charge state assignments ${ }^{47}$. Assignment of MS/MS spectra was performed using the SEQUEST algorithm ${ }^{48}$. Static modifications included TMT (229.16293 Da) on both the $\mathrm{N}$ terminus of peptides and lysine residues and carbamidomethylation of cysteine residues $(57.02146 \mathrm{Da})$. Peptide spectral matches were filtered to $1 \%$ FDR using the target-decoy strategy ${ }^{49}$, before being grouped into proteins which were then filtered to $1 \%$ FDR at the protein level as previously described ${ }^{47}$. All data analysis was performed using $\mathrm{R}$.

Western blot analysis. Cells were lysed in modified RIPA buffer $(50 \mathrm{mM}$ Tris- $\mathrm{HCl}$ $\mathrm{pH} 8,150 \mathrm{mM} \mathrm{NaCl}, 0.1 \%$ SDS, $0.5 \%$ sodium deoxycholate, $1 \%$ Triton X-100, $1 \mathrm{mM}$ EDTA, $1 \times$ protease inhibitor mixture (Roche Diagnostics), $1 \times$ PhosSTOP

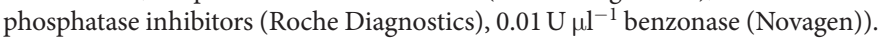
Cell lysate was then sonicated for five 30 -s pulses (with $30 \mathrm{~s}$ pauses) in a bath sonicator at $4{ }^{\circ} \mathrm{C}$ (Diagenode). Lysates were cleared of cell debris by centrifugation 
at $20,000 \mathrm{~g}$ for $5 \mathrm{~min}$. Westerns were performed with anti-Dnmt1 (1:500 dilution; Cell Signaling, 5119S), anti-Uhrf1 (1:200 dilution; Santa Cruz, sc-98817), anti-Dnmt3a (1:500 dilution; Santa Cruz Biotechnology, 20703), anti-Dnmt3b (1:1,000 dilution; Abcam, 13604), or anti- $\beta$-actin (1:10,000 dilution; Cell Signaling Technology, 5125S).

Data availability. The accession number for the raw data files for the RRBS analysis is NCBI Gene Expression Omnibus (GEO) GSE97995. The accession numbers for the raw data files for the RRBS analysis on female ES cell lines are NCBI GEO GSM1679872, GSM1679873, GSM1679874. The accession numbers for the raw data files for the RNA-seq analysis are NCBI GEO GSE97954 and GSE93103. The accession number for the raw data files for the genome-sequencing analysis is NCBI Sequence Read Archive (SRA) SRP102419. The accession number for the raw data files for the ChIP-seq analysis is NCBI GEO GSE98771. All other data are available from the corresponding author upon reasonable request.

27. Choi, J., Huebner, A. J. \& Hochedlinger, K. Derivation and long-term propagation of mouse embryonic stem cells. Protoc. Exch. http://dx.doi. org/10.1038/protex.2017.070 (2017).

28. Stadtfeld, M., Maherali, N., Borkent, M. \& Hochedlinger, K. A reprogrammable mouse strain from gene-targeted embryonic stem cells. Nat. Methods 7, 53-55 (2010).

29. Kaneda, M. et al. Essential role for de novo DNA methyltransferase Dnmt3a in paternal and maternal imprinting. Nature 429, 900-903 (2004).

30. Dodge, J. E. et al. Inactivation of Dnmt3b in mouse embryonic fibroblasts results in DNA hypomethylation, chromosomal instability, and spontaneous immortalization. J. Biol. Chem. 280, 17986-17991 (2005).

31. Boyle, P. et al. Gel-free multiplexed reduced representation bisulfite sequencing for large-scale DNA methylation profiling. Genome Biol. 13, R92 (2012).

32. Meissner, A. et al. Genome-scale DNA methylation maps of pluripotent and differentiated cells. Nature 454, 766-770 (2008).

33. Anders, S., Pyl, P. T. \& Huber, W. HTSeq-a Python framework to work with high-throughput sequencing data. Bioinformatics 31, 166-169 (2015)

34. Anders, S. et al. Count-based differential expression analysis of RNA sequencing data using R and Bioconductor. Nat. Protocols 8, 1765-1786 (2013).
35. Dobin, A. et al. STAR: ultrafast universal RNA-seq aligner. Bioinformatics $\mathbf{2 9}$, 15-21 (2013).

36. Robinson, M. D., McCarthy, D. J. \& Smyth, G. K. edgeR: a Bioconductor package for differential expression analysis of digital gene expression data. Bioinformatics 26, 139-140 (2010).

37. Keane, T. M. et al. Mouse genomic variation and its effect on phenotypes and gene regulation. Nature 477, 289-294 (2011).

38. Tarasov, A., Vilella, A. J., Cuppen, E., Nijman, I. J. \& Prins, P. Sambamba: fast processing of NGS alignment formats. Bioinformatics 31, 2032-2034 (2015).

39. Li, H. et al. The Sequence Alignment/Map format and SAMtools. Bioinformatics 25, 2078-2079 (2009)

40. Robinson, J. T. et al. Integrative genomics viewer. Nat. Biotechnol. 29, 24-26 (2011).

41. Treff, N. R. et al. Next generation sequencing-based comprehensive chromosome screening in mouse polar bodies, oocytes, and embryos. Biol. Reprod. 94, 76 (2016).

42. Xiao, A. et al. WSTF regulates the H2A.X DNA damage response via a novel tyrosine kinase activity. Nature 457, 57-62 (2009).

43. Langmead, B., Trapnell, C., Pop, M. \& Salzberg, S. L. Ultrafast and memoryefficient alignment of short DNA sequences to the human genome. Genome Biol. 10, R25 (2009).

44. Song, Q. \& Smith, A. D. Identifying dispersed epigenomic domains from ChIP-Seq data. Bioinformatics 27, 870-871 (2011).

45. McLean, C. Y. et al. GREAT improves functional interpretation of cis-regulatory regions. Nat. Biotechnol. 28, 495-501 (2010).

46. Eggan, K. et al. Hybrid vigor, fetal overgrowth, and viability of mice derived by nuclear cloning and tetraploid embryo complementation. Proc. Natl Acad. Sci. USA 98, 6209-6214 (2001).

47. Huttlin, E. L. et al. A tissue-specific atlas of mouse protein phosphorylation and expression. Cell 143, 1174-1189 (2010)

48. Eng, J. K., McCormack, A. L. \& Yates, J. R. An approach to correlate tandem mass spectral data of peptides with amino acid sequences in a protein database. J. Am. Soc. Mass Spectrom. 5, 976-989 (1994).

49. Elias, J. E. \& Gygi, S. P. Target-decoy search strategy for increased confidence in large-scale protein identifications by mass spectrometry. Nat. Methods $\mathbf{4}$, 207-214 (2007). 


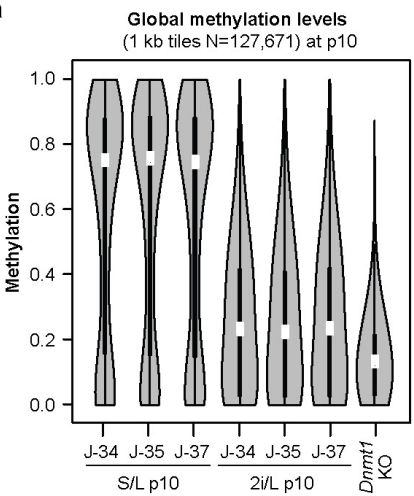

b

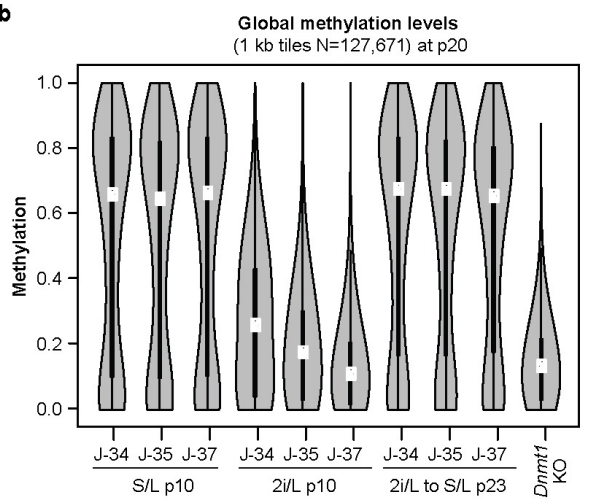

C Global methylation levels

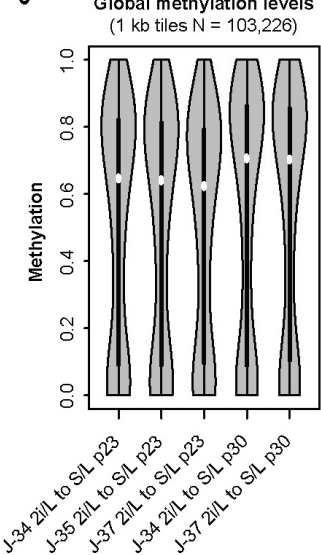

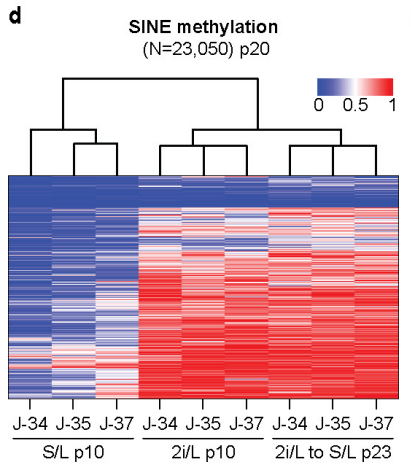

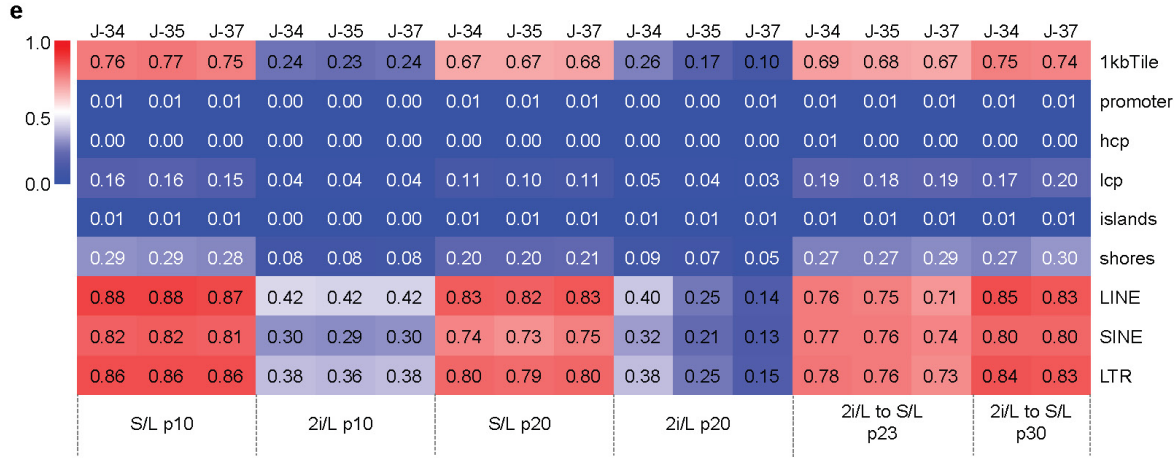

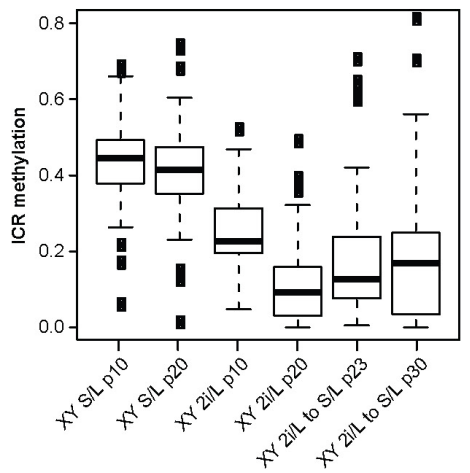

g

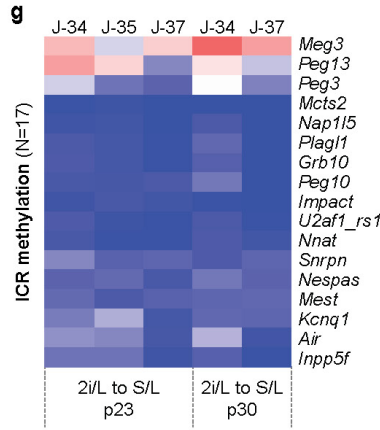

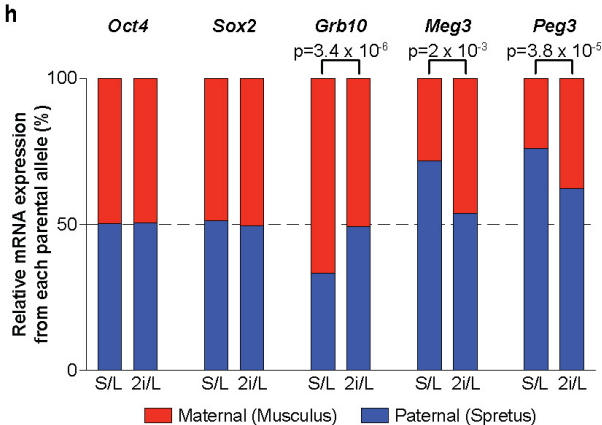

i

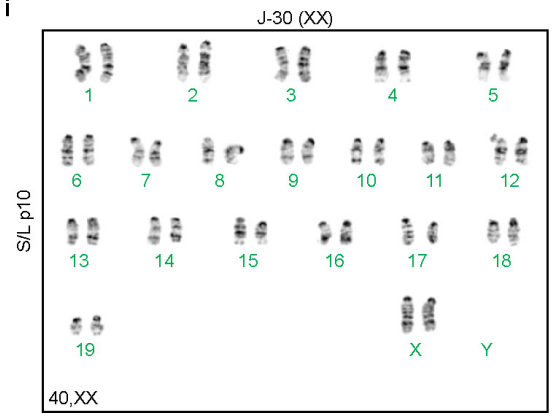

\begin{tabular}{|c|c|c|c|c|c|c|}
\hline \multicolumn{7}{|c|}{ J-31 $(X X)$} \\
\hline B & \multicolumn{2}{|c|}{30} & 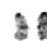 & \multicolumn{2}{|c|}{ 스 } & 68 \\
\hline 1 & \multicolumn{2}{|c|}{2} & 3 & \multicolumn{2}{|c|}{4} & 5 \\
\hline $\mathrm{A} g$ & \& & है 4 & 23 & 88 & 36 & ह \\
\hline 6 & 7 & 8 & 9 & 10 & 11 & 12 \\
\hline है & A & & & $2 \mathrm{~A}$ & $\hat{\alpha} z$ & \& $\mathrm{g}$ \\
\hline 13 & 14 & & & 16 & 17 & 18 \\
\hline 5 & & & & & 89 & \\
\hline 19 & & & & & $x$ & $Y$ \\
\hline $40, x \times$ & & & & & & \\
\hline
\end{tabular}

Extended Data Figure 1 | Effects of different culture conditions on DNA methylation in ES cells. a-c, Violin plots showing global methylation levels of ES cells at p10 (a), p20 (b) and after being placed back into S/L at p23 and p30 (c) by RRBS. Hypomethylated Dnmt 1 knockout (KO) ES cells were included as control. White dots denote median value. d, Heat map of DNA methylation levels at SINE elements in ES cells. e, Median methylation levels of different genomic elements in the indicated conditions. f, Box plot showing methylation levels of ICRs cultured in each condition. Thick horizontal lines represent median values. Box shows the interquartile range of the data. Whiskers extend to the most extreme data point that is no more than 1.5 times the interquartile range. Outliers beyond this range are shown as points. $\mathrm{g}$, ICR methylation levels after being re-exposed to $\mathrm{S} / \mathrm{L}$ for the indicated passages. $\mathbf{h}$, RNA sequencing was performed on a F1 Mus musculus $\times$ Mus spretus stem-cell line cultured in $2 \mathrm{i} / \mathrm{L}$ for 6 passages. Normalized read counts of mRNA transcripts expressed from the paternal or maternal alleles are shown based on multiple allele-discriminating single nucleotide polymorphisms (SNPs) for each gene (6 or more SNPs per gene). Statistical analysis (two-tailed $t$-tests) for allelic biases of the SNPs is shown. i, Karyotyping results from two female ES cell lines cultured in S/L for 10 passages. 


\section{RESEARCH LETTER}

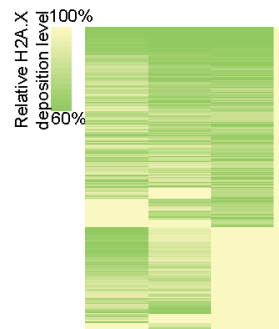

XXX S/L XY 2i/L XY Zi/L XY S/L to $S / L$

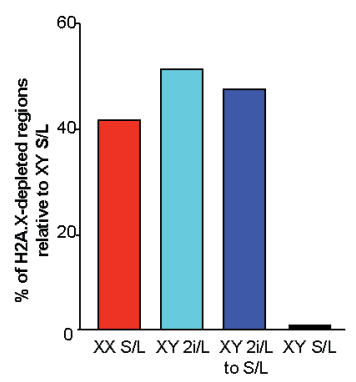

c

Relative H2A.X deposition level (XY 2i/L)/(XY S/L)
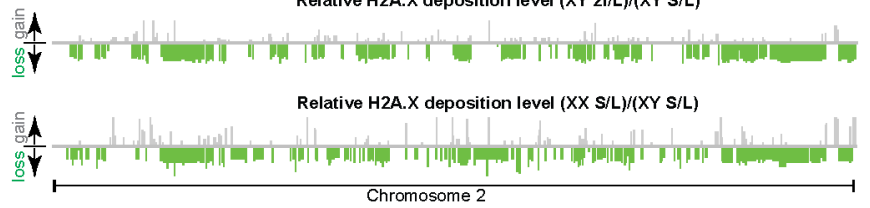

d

e

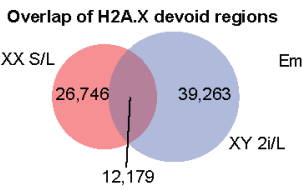
Mesoderm morphogenesis morphogenesis development Gastrulation $-\log _{10} \stackrel{50}{50} 100$

Extended Data Figure 2 | Effects of prolonged 2i/L culture on H2A.X deposition in ES cells. a, Heat map showing global H2A.X deposition loss relative to male ES cells cultured in S/L. Three ES cell lines per condition (male S/L, male 2i/L, female S/L) at p10 and two ES cell lines (male 2i/L to $\mathrm{S} / \mathrm{L}$ ) at p30 were analysed (see Fig. 1a). b, Bar graph quantifying the percentage of H2A.X-depleted regions relative to male ES cells cultured in $\mathrm{S} / \mathrm{L}$. Each bar represents median values of biological replicates of samples in a. c, Relative H2A.X deposition on a representative chromosome. Relative losses (green bars) or gains (grey bars) of H2A.X are mapped to their location on chromosome 2. d, Overlap of devoid H2A.X regions common to both female ES cells cultured in S/L and male cells cultured in $2 \mathrm{i} / \mathrm{L}$. e, H2A.X-devoid regions present in the overlap identified in $\mathbf{d}$ were significantly enriched for genes involved in the listed developmental pathways. The GREAT bioinformatic database was used to bin the genes identified into transcriptional networks. 


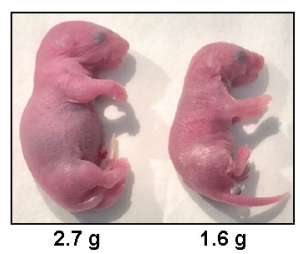

b

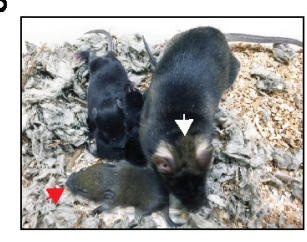

c

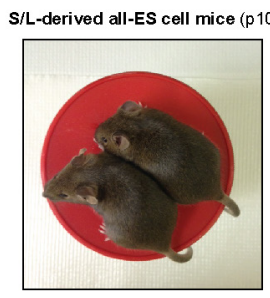

d

e

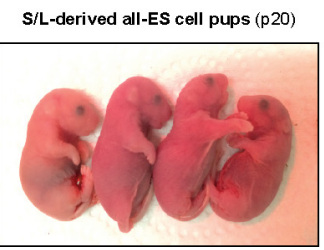

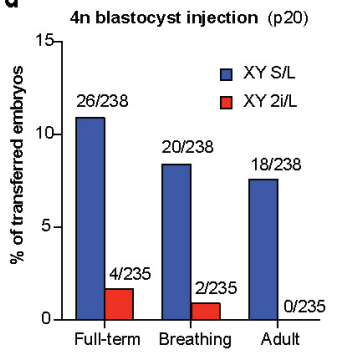

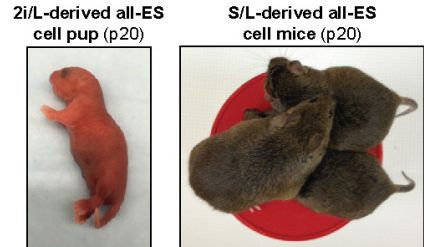

Extended Data Figure 3 Developmental potential of ES cells cultured in $S / L$ and $2 i / L$. a, Representative image of a chimaeric pup derived from female ES cells cultured in S/L (left) compared to a pup obtained through natural mating (right). Body weight of each pup was shown below. b, Representative image of a low-grade adult chimaera with patches of agouti hairs generated using female ES cells in S/L (white arrow). Her agouti germline offspring (red arrowhead) was generated by crossing her to a C57B6 wild-type mouse. c, Representative image of all-ES cell adult mice generated from male ES cells cultured in S/L for 10 passages. d, Bar graphs showing percentages of transferred $4 n$ blastocysts that survived to birth (full-term), established regular breathing or survived past 5 weeks (adult) using male ES cells grown in either $\mathrm{S} / \mathrm{L}$ (blue bars) or $2 \mathrm{i} / \mathrm{L}$ (red bars) until p20. The number of animals obtained per total number of transferred embryos is shown. e, Representative images of all-ES cell neonates produced from male ES cells cultured in S/L (left) and 2i/L (middle) at p20. Representative image of all-ES cell adult mice generated from ES cells cultured in S/L at p20 (right). 


\section{RESEARCH LETTER}

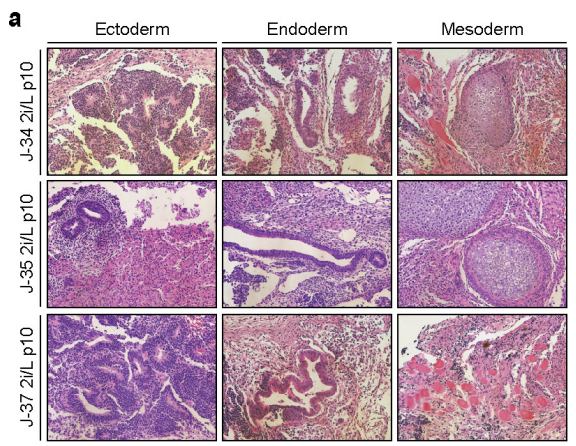

b
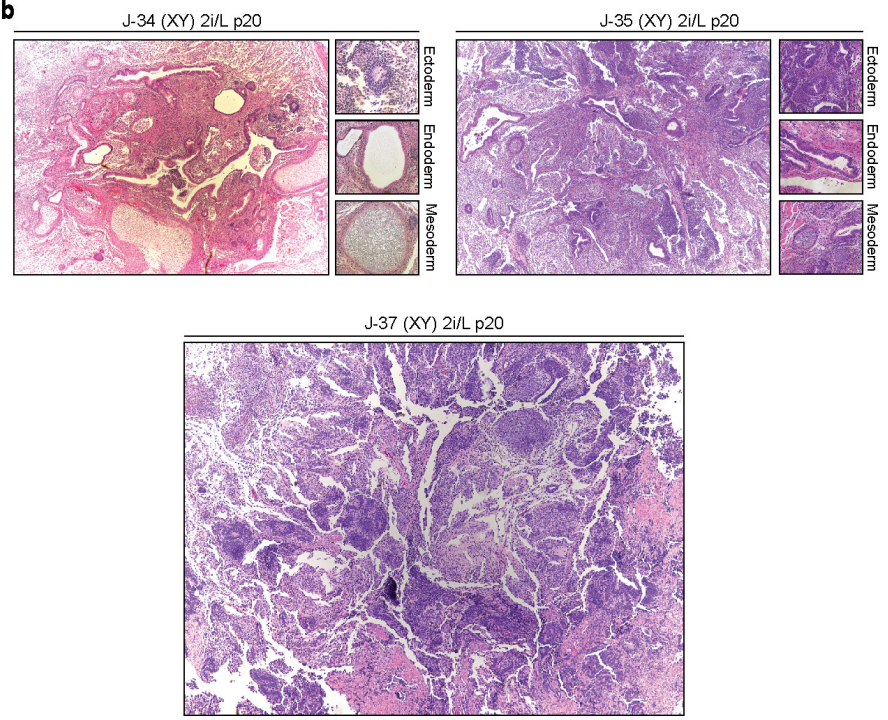

Extended Data Figure 4 | Potential of male ES cells cultured in $2 \mathrm{i} / \mathrm{L}$ to produce teratomas. a, Karyotype analysis of male ES cell lines grown in $2 \mathrm{i} / \mathrm{L}$ at $\mathrm{p} 10$. b, Representative images of teratomas produced with three male ES cell lines grown in $2 \mathrm{i} / \mathrm{L}$ at p20 with depiction of germ layer differentiation where detectable. 
a

Array Comparative Genomic Hybridization (aCGH)
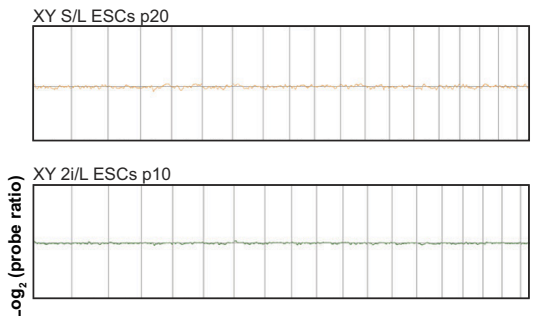

XY 2i/L ESCs 20

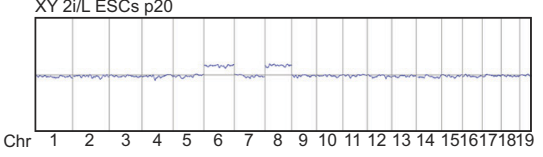

b

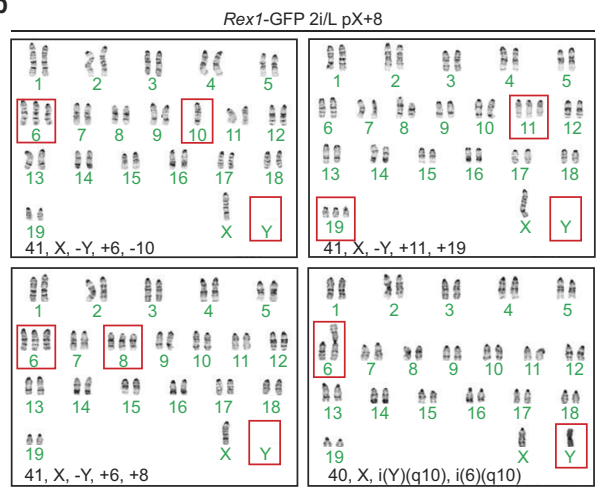

d

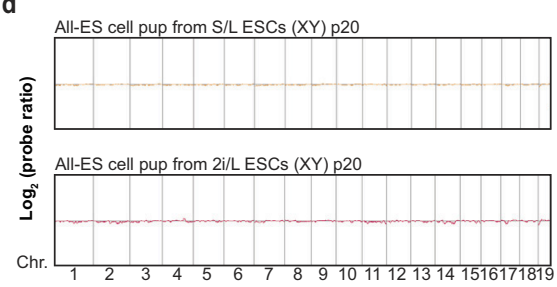

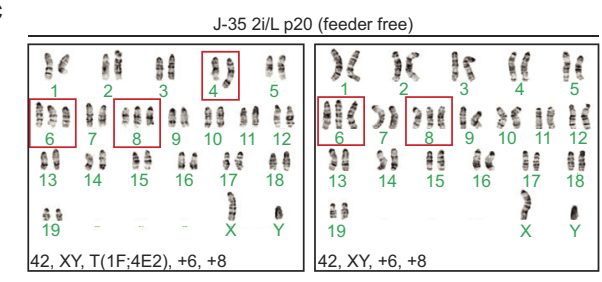

ICR methylation $(\mathrm{N}=17)$

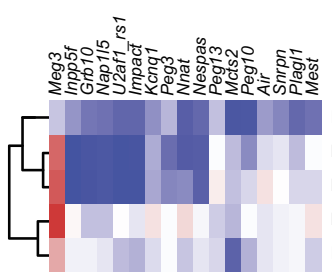

$\operatorname{ESCs}(X Y, 2 i / L p 10)$ Keratinocytes (wild-type)

$\operatorname{ESCs}(X Y, S / L p 10)$ Keratinocytes \#1 (from XY ESC, 2i/L p10)

Extended Data Figure 5 | Effects of prolonged 2i/L culture on chromosomal stability in ES cells. a, aCGH analysis of the J-35 ES cell line cultured in $\mathrm{S} / \mathrm{L}$ or $2 \mathrm{i} / \mathrm{L}$ condition for the indicated passage numbers. b, Karyotype analysis of Rex1-GFP ES cell line grown in 2i/L for 8 passages after receiving the line. Red boxes indicate abnormal chromosomes detected. c, Karyotype analysis of J35 ES cell line grown in $2 \mathrm{i} / \mathrm{L}$ in the absence of a layer of MEF feeder cells for 16 passages (p20).
Red boxes indicate abnormal chromosomes detected. d, aCGH analysis on DNA isolated from a newborn pup generated by $4 n$ blastocyst injection using ES cells cultured in S/L or $2 \mathrm{i} / \mathrm{L}$ at 20 passages. e, Combined dendrogram and heat map depicting ICR methylation levels in ES cells ( $n=3$ biological replicates) and keratinocytes using RRBS analysis. ' $2 \mathrm{i} / \mathrm{L}$ keratinocytes' were explanted from chimaeric adult mice derived from male 2i/L ES cell p10 and purified by drug selection (G418). 
a

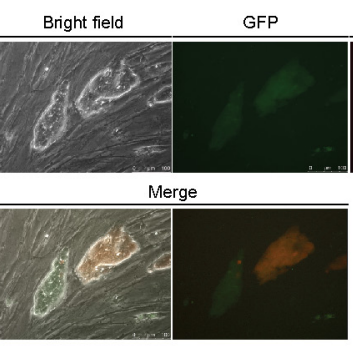

b
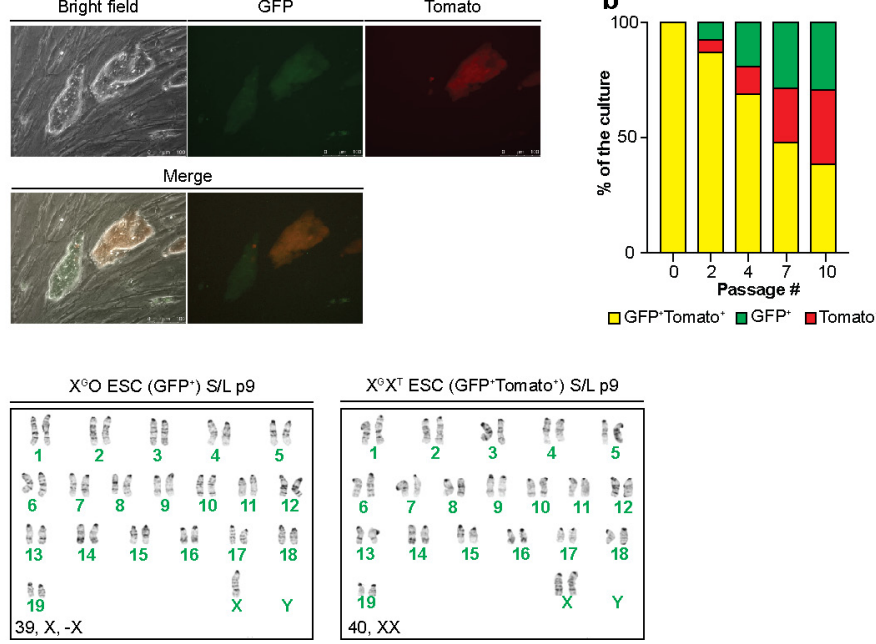

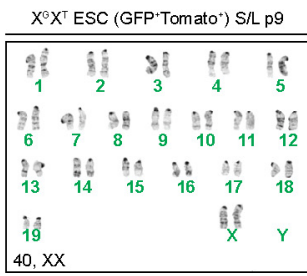

Extended Data Figure 6 | Characterization of the $X^{G} X^{T}$ ES cell line. a, Fluorescence microscopic images of $\mathrm{X}^{\mathrm{G}} \mathrm{X}^{\mathrm{T}} \mathrm{ES}$ cells. GFP/tdTomato double-positive cells (yellow cells in merge 1 and 2) indicate two active $\mathrm{X}$ chromosomes while $\mathrm{GFP}^{+}$Tomato $^{-}$colony depicts cells that lost one of the X chromosomes. $\mathbf{b}$, GFP/tdTomato double-positive $\mathrm{X}^{\mathrm{G}} \mathrm{X}^{\mathrm{T}} \mathrm{ES}$ cells were sorted at passage 5 and plated in S/L (p6) before measuring the percentage of double-positive and single-positive cells at p8, p10, p13 and p16 using flow cytometry. c, Karyotype analysis of undifferentiated, $\mathrm{X}^{\mathrm{G}} \mathrm{O}$ (GFP-positive) or $\mathrm{X}^{\mathrm{T}} \mathrm{O}$ (tdTomato-positive) ES cells (left) and GFP/ tdTomato double-positive $\mathrm{X}^{\mathrm{G}} \mathrm{X}^{\mathrm{T}} \mathrm{ES}$ cells (right). GFP/tdTomato doublepositive $\mathrm{X}^{\mathrm{G}} \mathrm{X}^{\mathrm{T}} \mathrm{ES}$ cells were sorted at passage 5 and maintained in $\mathrm{S} / \mathrm{L}$ for 9 passages before analysis. This result confirms that the progressive loss of the GFP or TdTomato signal was due to the loss of an X chromosome, and not X-chromosome inactivation due to differentiation. 


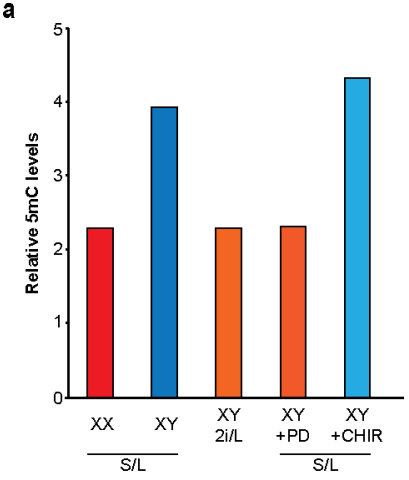

C

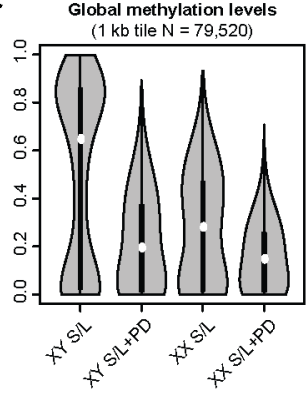

Extended Data Figure 7 | Consequences of PD0325901 and CHIR99021 treatment on DNA methylation. a, Global methylation levels of were analysed by $5 \mathrm{mC}$ dot blot analysis for male and female ES cell lines cultured in the indicated conditions for 6 passages.

b, Median methylation levels at the indicated genetic elements following inhibition or loss of Mek1/2 or Gsk3 $\alpha / \beta$, respectively. c, Violin plots b

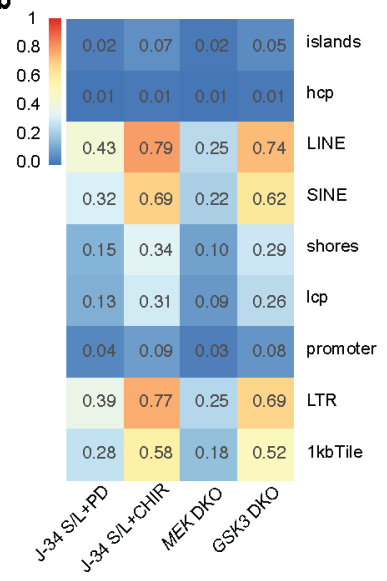

d

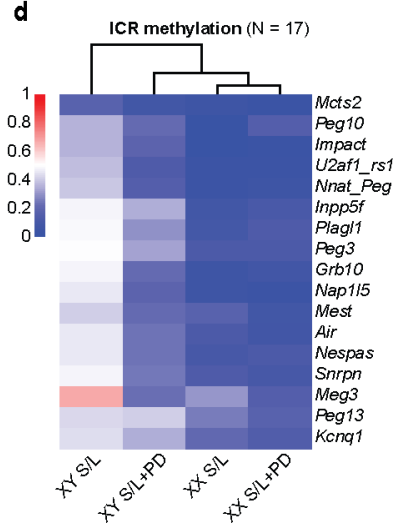

showing global methylation levels of male and female ES cells cultured in S/L for 6 passages and then cultured for an additional 3 passages in S/L supplemented with PD0325901 (PD). White boxes indicate median methylation levels. d, Heat map and dendrogram of ICR methylation levels in male and female ES cells shown in $\mathbf{c}$. 


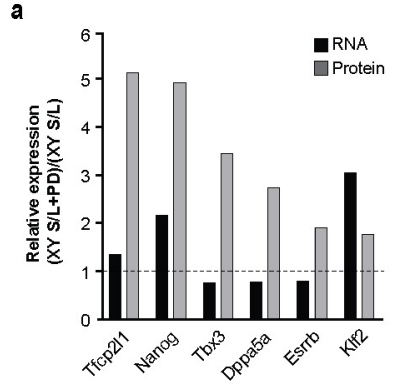

b

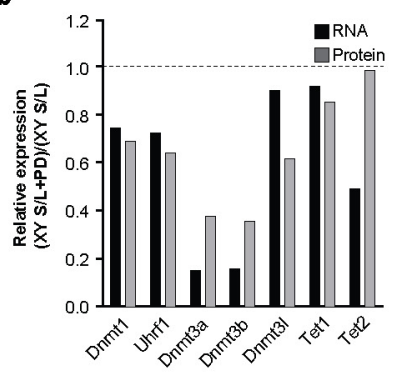

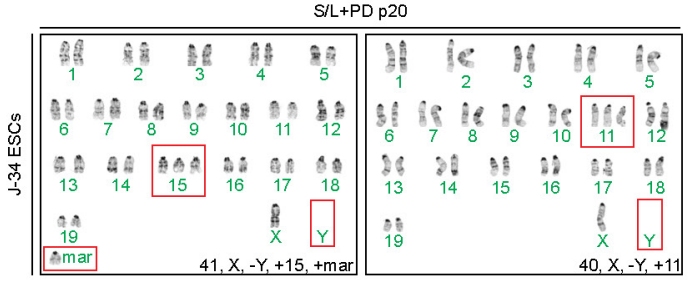

d

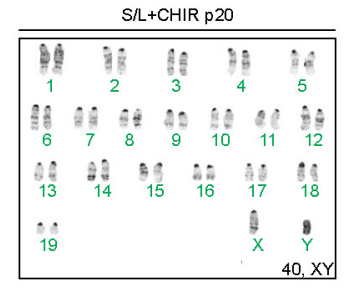

e

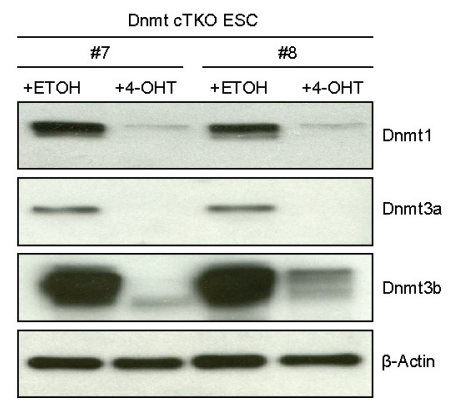

f
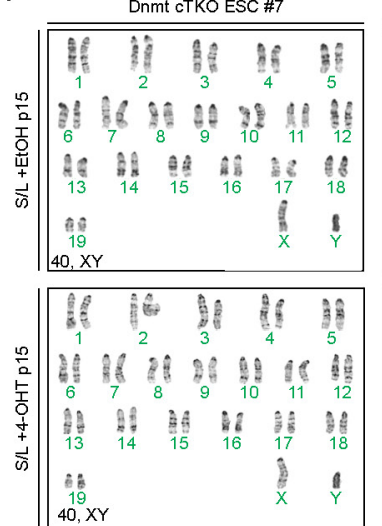

Dnmt cTKO ESC \#8

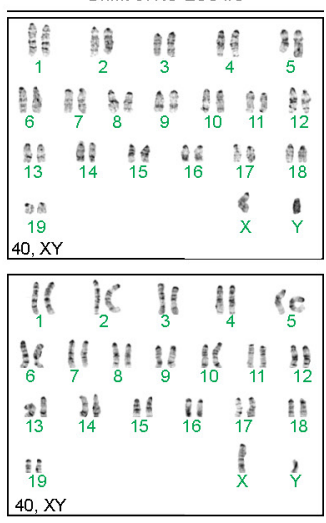

cultured in either S/L + PD (c) or S/L + CHIR (d) for additional 16 passages. Red boxes indicate abnormal chromosomes detected. e, Western blot analysis of a Dnmt conditional triple-knockout (cTKO) ES cell line for Dnmt1, Dnmt3a and Dnmt3b after treatment with either 4-OHT or ethanol (EtOH) for one passage. f, Karyotype analysis of a Dnmt conditional triple-knockout ES cell line that was treated with either 4-OHT or ethanol for one passage and subsequently cultured for 15 passages in S/L. 


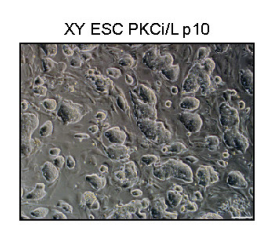

XY ESC a2/L $p 10$

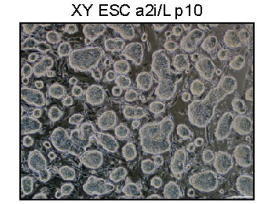

e

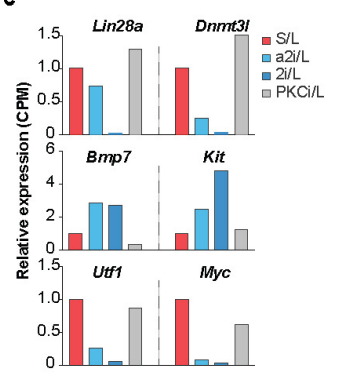

h

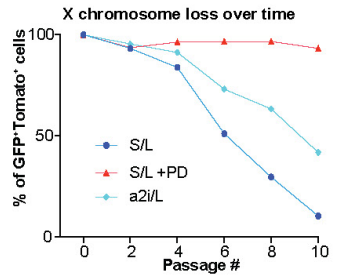

i

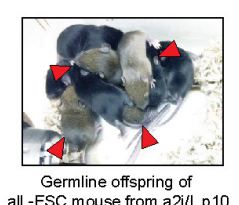

b

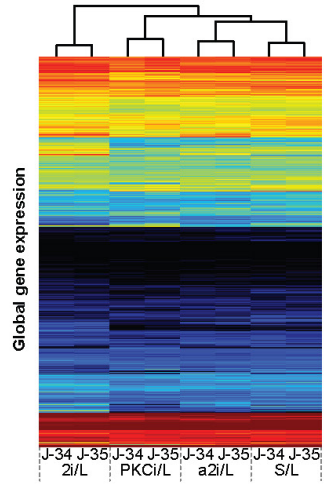

f Global methylation levels

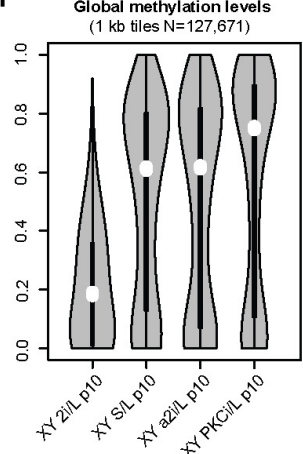

j

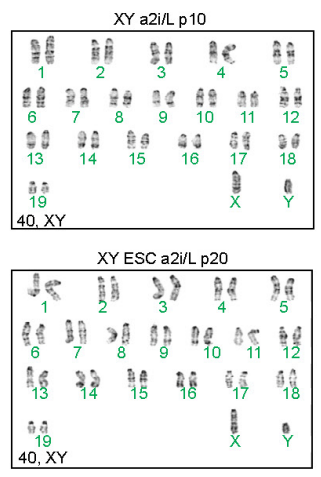

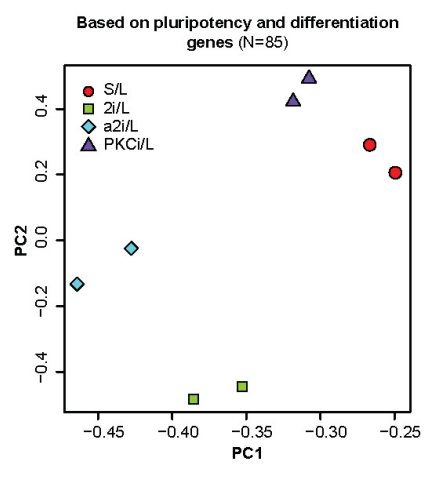

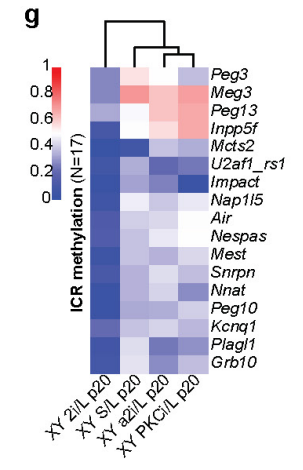

k
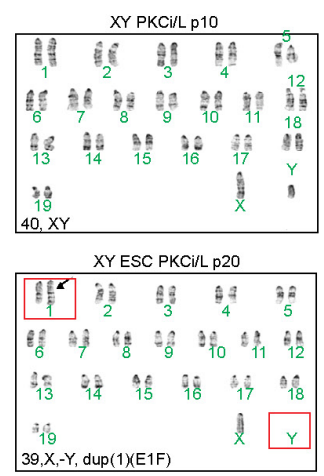

d

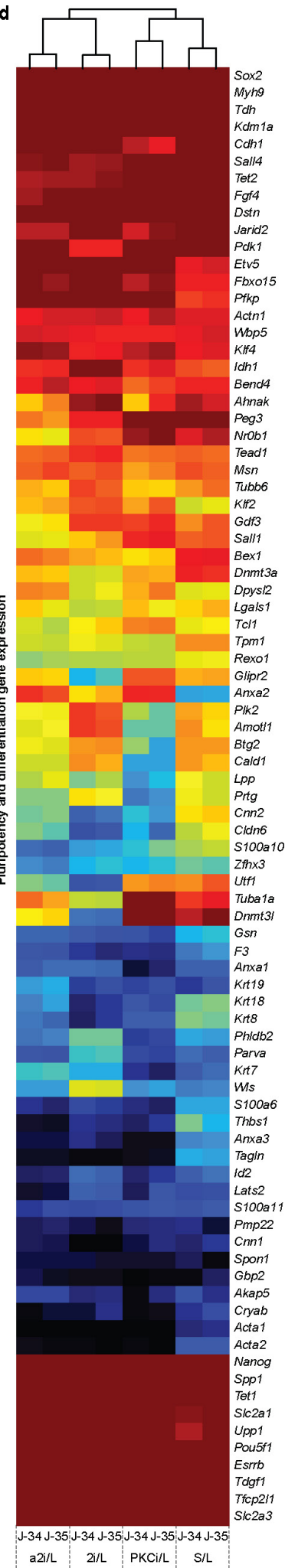

\begin{tabular}{l|l|l|l|l}
\hline$a 2 i / L$ & $2 i / L$ & $P K C i / L$ & $S / L$
\end{tabular}
Extended Data Figure 9 | Characterization of alternative culture conditions for ES cells. a, Representative images of XY ES cells cultured in the indicated conditions. $\mathbf{b}$, Heat map and dendrogram of global gene expression levels in male ES cells cultured in the indicated conditions. c, Principal component analysis of ES cells cultured in S/L, 2i/L, a2i/L or $\mathrm{PKCi} / \mathrm{L}$ using a previously published set of known pluripotency and differentiation genes ${ }^{23}$. Two male ES cell lines maintained in S/L for 4 passages were switched to each condition and cultured for additional 6 passages (final passage 10). d, Heat map and dendrogram showing expression levels of pluripotency and developmental genes ${ }^{23}$ in male ES cell lines cultured in the indicated conditions. e, Relative expression levels of transcripts associated with naive pluripotency in d. f, Violin plots of global methylation levels in the indicated ES cell lines. White dots, median value. $\mathbf{g}$, Heat map and dendrogram of ICR methylation levels in the indicated ES cells lines. h, Flow-cytometric analysis of GFP and TdTomato after culturing $X^{G} X^{T} E S$ cells in $S / L, S / L+P D$ and a2i/L for the indicated passages. i, Agouti germline offspring (red arrows), obtained from across between an all-ES cell male generated from p10 a2i/L-cultured ES cells and a C57B6 female. j, k, Karyotype analysis of male ES cells cultured in a2i/L for 6 or 16 passages $(\mathbf{j})$, or PKCi/L for 6 or 16 passages $(\mathbf{k})$. Red boxes indicate abnormal chromosomes detected. 


\section{RESEARCH LETTER}

a

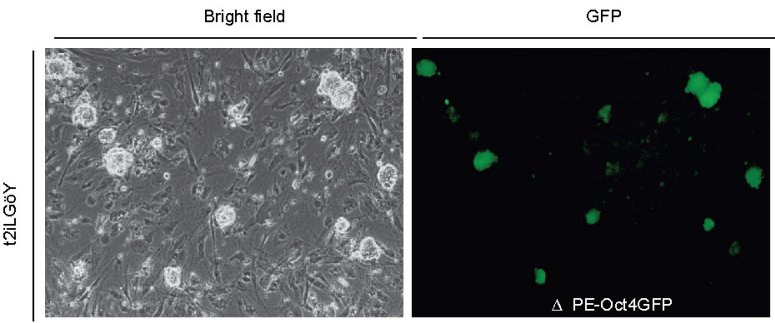

b
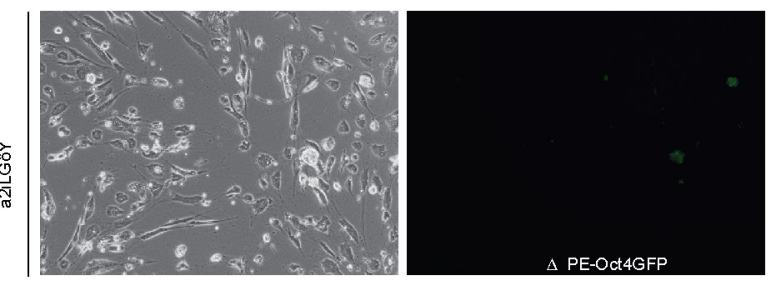

C

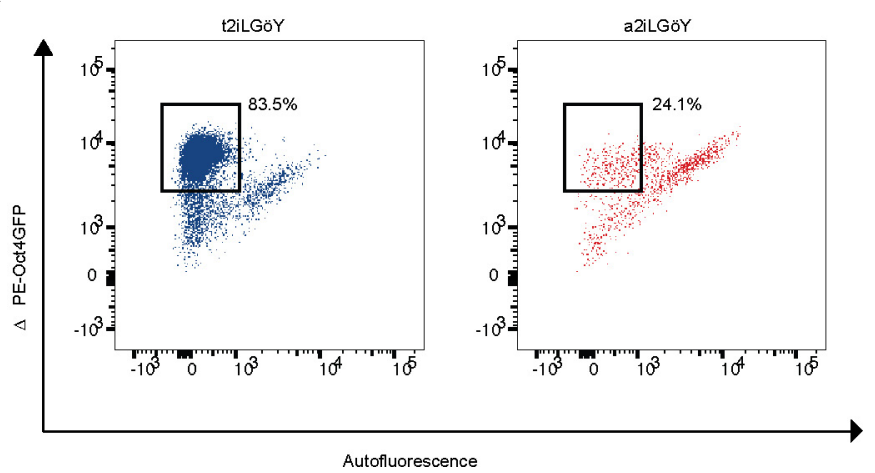

Extended Data Figure 10 | Replacement of MEK1/2 inhibitor with SRC inhibitor using naive human ES cells. a, Representative image of human ES cells carrying a naive-specific OCT4-eGFP reporter $(\triangle \mathrm{PE}-\mathrm{OCT} 4-\mathrm{eGFP})^{24}$ cultured in the presence of small molecule inhibitors of the ROCK, MEK1/2, GSK3 and PKC kinases (t2iLGöY) ${ }^{25}$. Bright field image (left) and GFP expression (right). b, Representative image of $\triangle$ PE-OCT4-eGFP human ES cells cultured in the presence of small molecule inhibitors of the ROCK, SRC, GSK3 and PKC kinases (a2iLGöY). Bright field image (left) and GFP expression (right). c, Flowcytometric analysis of eGFP expression in $\triangle \mathrm{PE}-\mathrm{Oct} 4 \mathrm{GFP}$ human ES cells cultured in t2iLGöY or a2iLGöY. 